Article

\title{
Ipomoea pes-caprae IpASR Improves Salinity and Drought Tolerance in Transgenic Escherichia coli and Arabidopsis
}

\author{
Jie-Xuan Zheng ${ }^{1,2,+}$, Hui Zhang ${ }^{1,2,+}$, Hua-Xiang Su ${ }^{1,2}$, Kuai-Fei Xia ${ }^{1,3}$, Shu-Guang Jian ${ }^{3}$ and \\ Mei Zhang $1,3, *$ \\ 1 Key Laboratory of South China Agricultural Plant Molecular Analysis and Genetic Improvement, \\ South China Botanical Garden, Chinese Academy of Sciences, Guangzhou 510650, China; \\ zhengjiexuan16@mails.ucas.ac.cn (J.-X.Z.); zhanghuir@mails.ucas.ac.cn (H.Z.); \\ suhuaxiang17@mails.ucas.ac.cn (H.-X.S.); xiakuaifei@scbg.ac.cn (K.-F.X.) \\ 2 University of the Chinese Academy of Sciences, Beijing 100039, China \\ 3 Key Laboratory of Applied Botany, South China Botanical Garden, Chinese Academy of Sciences, \\ Guangzhou 510650, China; jiansg@scbg.ac.cn \\ * Correspondence: zhangmei@scbg.ac.cn \\ + These authors contributed equally to this work.
}

Received: 24 June 2018; Accepted: 30 July 2018; Published: 1 August 2018

\begin{abstract}
Ipomoea pes-caprae L. is an extremophile halophyte with strong adaptability to seawater and drought. It is widely used in the ecological restoration of coastal areas or degraded islands in tropical and subtropical regions. In this study, a new abscisic acid, stress and ripening (ASR) gene, IpASR, was reported, and is mainly associated with biological functions involved in salt and drought tolerance. Sequence analysis of IpASR showed that this protein contains an ABA/WDS (abscisic acid/water deficit stress) domain, which is a common feature of all plant ASR members. Overexpression of IpASR improved Escherichia coli growth performance compared with the control under abiotic stress treatment. The transgenic overexpressing IpASR Arabidopsis showed higher tolerance to salt and drought stress than the wild type and lower accumulation of hydrogen peroxide $\left(\mathrm{H}_{2} \mathrm{O}_{2}\right)$ and superoxide $\left(\mathrm{O}_{2}{ }^{-}\right)$accompanied by increased antioxidant enzyme activity in vivo. IpASR exhibits transcription factor's activity. Therefore, the overexpression of IpASR in Arabidopsis is supposed to influence the expression of some genes involved in anti-oxidative and abiotic stresses. The results indicate that IpASR is involved in the plant response to salt and drought and probably acts as a reactive oxygen species scavenger or transcription factor, and therefore influences physiological processes associated with various abiotic stresses in plants.
\end{abstract}

Keywords: Ipomoea pes-caprae L.; IpASR; salt; drought

\section{Introduction}

Due to the immobility of plants, their growth and development is frequently influenced by external environmental signals, particularly by certain biotic or abiotic stresses. The response of plants to stress mainly involves the following steps: The perception and transduction of stress signals and the induction of the expression or synthesis of the response gene or protein, resulting in cellular changes in physiological and biological characteristics, eventually decreasing the damage caused by stress [1]. Among these responses, the regulation of the expression of stress-related genes is a vital step for the survival or tolerance of plants in adverse conditions. The isolation and characterization of stress-tolerance genes provides a foundation for the genetic improvement of plants and crops [2]. ASR (abscisic acid, stress, ripening-induced) genes are plant-specific small gene families that are mostly 
involved in the response to abiotic (mainly drought and salinity) stresses and fruit ripening [3]. The adaptation of many plants and crops to stressful environments in the context of environmental degradation and climate change is being increasingly studied in an attempt to improve stress tolerance and isolate candidate stress-related genes for plant genetic engineering.

ASR proteins occur in many plant species and belong to a family of hydrophilic proteins that have important roles in the response to abiotic stresses. All ASRs harbor the ABA/WDS (abscisic acid/water deficit stress) domain (Pfam entry PF02496) as a common denominator, indicating that they might have similar biological functions in the response of plants to dehydration or the abscisic acid (ABA) signaling pathway [4]. Since ASRs may not only function as transcription factors, but also as intrinsically unstructured chaperones possessing similar plant LEA (late embryogenesis abundant) protein characteristics, some reporters classified as ASR proteins have been placed into the LEA protein group because of their small size, physicochemical properties, and participation in stress pathways [5]. While with a distinguished feature from LEA family, a large multigene family, the ASR family is obviously absent from Brassicaceae plants (including Arabidopsis), and usually comprise few members in other plants. The first member of $A S R$, tomato Asr1, was identified by screening a tomato fruit cDNA library using a cDNA differential hybridization approach, with accumulated transcripts observed in the ripening fruit, water-stressed leaves, or under ABA treatment, hence its name [6]. Following this, a fairly large number of $A S R$ orthologs were characterized from many plant species along with the advance of the Plant Genome Project [7-10].

Plant-specific ASR genes are typically upregulated by a wide range of factors, including drought, cold, salt, ABA, and injury, in addition to the plant response to developmental and environmental signals. Drought and salinity are two common abiotic stressors that often challenge plant growth and development, thereafter triggering complex multicomponent signaling pathways to restore cellular homeostasis and promote plant survival [11]. Recent studies in many plants suggest that different ASRs could elevate the tolerance of transgenic plants to high salinity and dehydration. For instance, the MpAsr from Musa paradisiaca (banana) conferred improved osmotic tolerance to transgenic Arabidopsis [12]. Salicornia brachiata is an extreme halophyte that can survive in salty marshes in coastal areas. SbASR-1 from S. brachiata conferred enhanced salinity and drought endurance in transgenic tobacco and groundnut $[13,14]$. Suaeda liaotungensis is also an extreme halophyte that is mainly distributed on saline and alkaline lands in Heilongjiang province and the Inner Mongolia province in China. The SlASR from S. liaotungensis also elevates salinity and drought tolerance in transgenic plants when overexpressed in Arabidopsis [15]. The gramineous plants Setaria italica and Brachypodium distachyon are both drought resistant plants. SiASR4 [16] from S. italica and BdASR1 [9] from $B$. distachyon both enhanced salt and drought tolerance when they were separately overexpressed in Arabidopsis or tobacco.

Ipomoea pes-caprae L. (Convolvulaceae) is a wild perennial lianaceous herb that naturally occurs in seashore areas of tropical or subtropical regions worldwide. It exhibits high nutrition uptake and utilization efficiency and strong resistance to salt and drought, and hence has been used as the first "green barrier" for sand fixation, island-greening, and the ecological restoration of coastal regions and islands and reef construction [17]. As evidenced by its habitat, I. pes-caprae is also an extreme halophyte, which is considered to be the best resource for characterizing stress-tolerance genes and promoters [18]. These are then ultimately introduced into non-halophytic crop species to improve their performance under saline conditions [19]. In this study, we mainly focused on the characterization of IpASR from I. pes-capraei, and performed transgenic assays in Escherichia coli and Arabidopsis thaliana to functionally validate this gene under salt/drought and other abiotic stress conditions. 


\section{Results}

\subsection{IpASR Encodes a Protein with an ABA/WDS Domain}

IpASR was obtained by screening the I. pes-caprae full-length cDNA library (data not published). The full-length cDNA of IpASR is 962 bp with a 648 bp open reading frame (ORF) encoding a protein of 215 amino acids. The sequences of IpASR cDNA and protein are listed in Supplementary Materials Table S1. PCR amplification of genomic sequence of IpASR indicated that this gene contains a $192 \mathrm{bp}$ intron (Supplementary Materials Table S1). The ProtParam-calculated molecular weight of IpASR was $24.57 \mathrm{kDa}$ with a theoretical isoelectric point (pI) of 5.42. Glutamic acid was the most abundant amino acid in the IpASR protein sequence (encompassing $17.67 \%$ of the total number of amino acids), followed by lysine $(11.63 \%)$, and glycine $(11.16 \%)$. Furthermore, the IpASR sequence carried 49 negatively charged (aspartic acid and glutamic acid) and 31 positively charged (arginine and lysine) residues. The instability index (II) of IpASR was also assessed by ProtParam, which classified this protein as stable with an instability index (II) score of $36.82(<40)$. The negative value $(-1.637)$ of the grand average of hydropathicity (GRAVY) indicated that the IpASR protein was hydrophilic. The PHYRE ${ }^{2}$ program suggested that $77 \%$ of the amino acid residues of IpASR were disordered, indicating that IpASR is an intrinsically disordered protein (IDP). The properties of the deduced IpASR are listed in Table 1.

Table 1. The physical and chemical properties of the IpASR protein.

\begin{tabular}{cc}
\hline Physical and Chemical Properties & IpASR \\
\hline No. of amino acids & 215 \\
Molecular weight $(\mathrm{kDa})$ & 24.57 \\
Theoretical pI & 5.42 \\
Total no. of negatively charged residues (D + E) & 49 \\
Total no. of positively charged residues (R + K) & 31 \\
Grand average of hydropathicity (GRAVY) & -1.637 \\
instability index (II) & 36.82 \\
Proportion of disordered amino acids (\%) & 77 \\
\hline
\end{tabular}

SmartBLAST analysis with the amino acid sequence of IpASR showed that it is highly homologous to InASR from Ipomoea nil, SdASR-1 from S. brachiata, and SlASR from S. liaotungensis (Figure 1). IpASR contains a highly conserved ABA/WDS functional domain (amino acids 128-184). At the N-terminal, a consensus sequence containing six His residues in IpASR was identified, which is speculated as a metal binding His-rich motif. There are also two Ala-rich motifs at the N-terminal region, one site for a cryptic $N$-myristoylation, and a putative nuclear localization signal (NLS), which implied that IpASR belongs to nucleoproteins (Figure 1). 


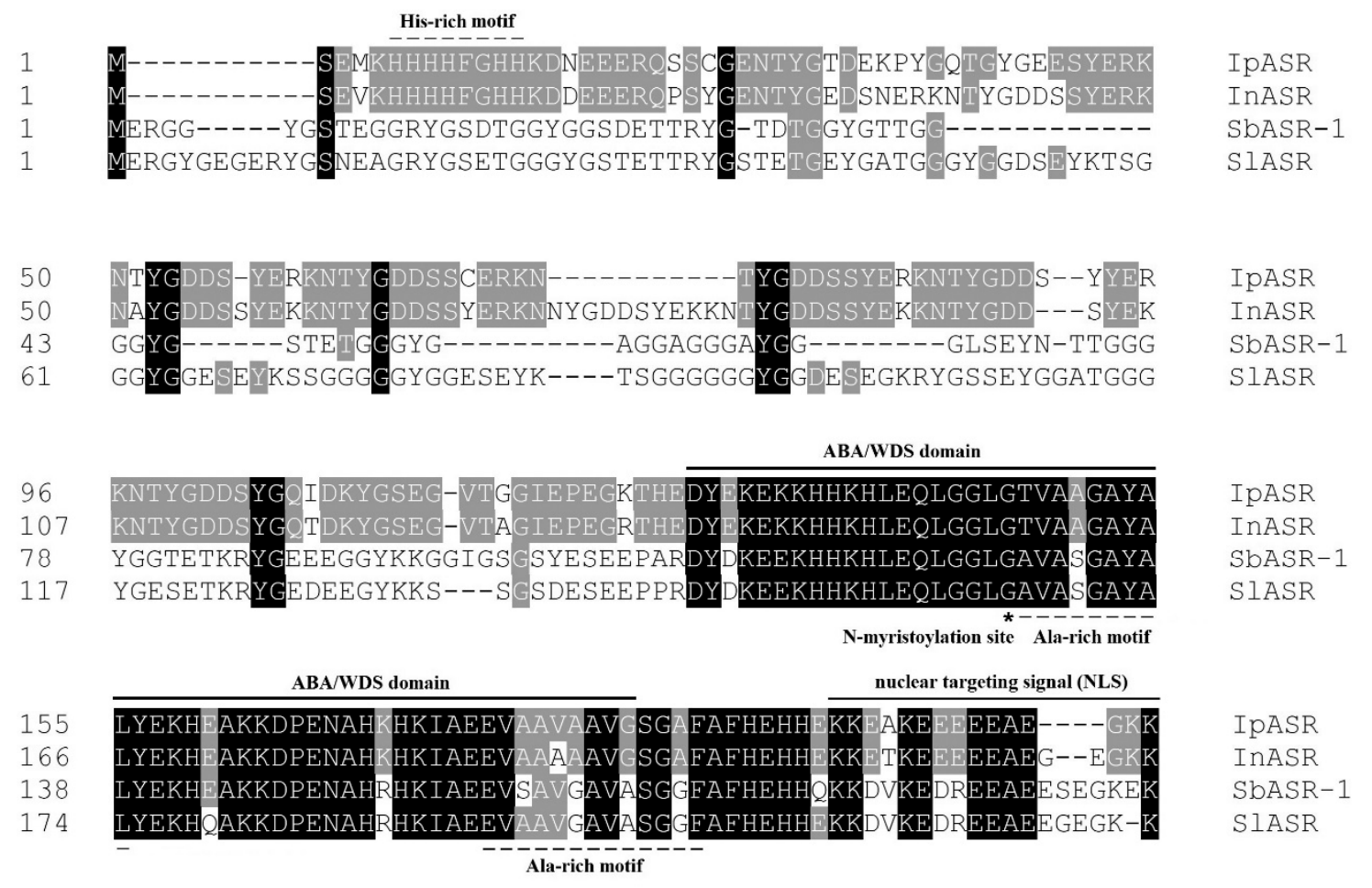

$\begin{array}{lll}211 & \overline{\text { KHHFF }} & \text { IpASR } \\ 224 & \text { KHHFF } & \text { InASR } \\ 198 & \text { KHHFF } & \text { SbASR-1 } \\ 233 & \text { KHHFF } & \text { SIASR }\end{array}$

Figure 1. Comparison of IpASR from I. pes-caprae with abscisic acid, stress and ripening (ASR) proteins from other plant species; I. nil InASR (BAF46301.1), S. brachiata SbASR-1 (ACI15208.1), and S. liaotungensis SIASR (AGZ20206.1). The abscisic acid/water deficit stress (ABA/WDS) domain, one His-rich motif, two Ala-rich motifs, and one cryptic $N$-myristoylation site were marked.

\subsection{Phylogenetic Analysis}

The deduced amino acid sequences of IpASR1 and the other 41 ASR members from other plant species were studied using phylogenetic analysis (Figure 2). Based on the amino acid sequences, IpASR shared maximum identity with the ASR proteins of morning glory InASR (Accession no. BAF46301.1) and Calystegia soldanella CsoASR (Accession no. BAB19963.1), which both belong to Convolvulaceae. However, the phylogenetic tree did not reflect the evolutionary relationships between glycophyte and halophyte or monocot and dicot. 


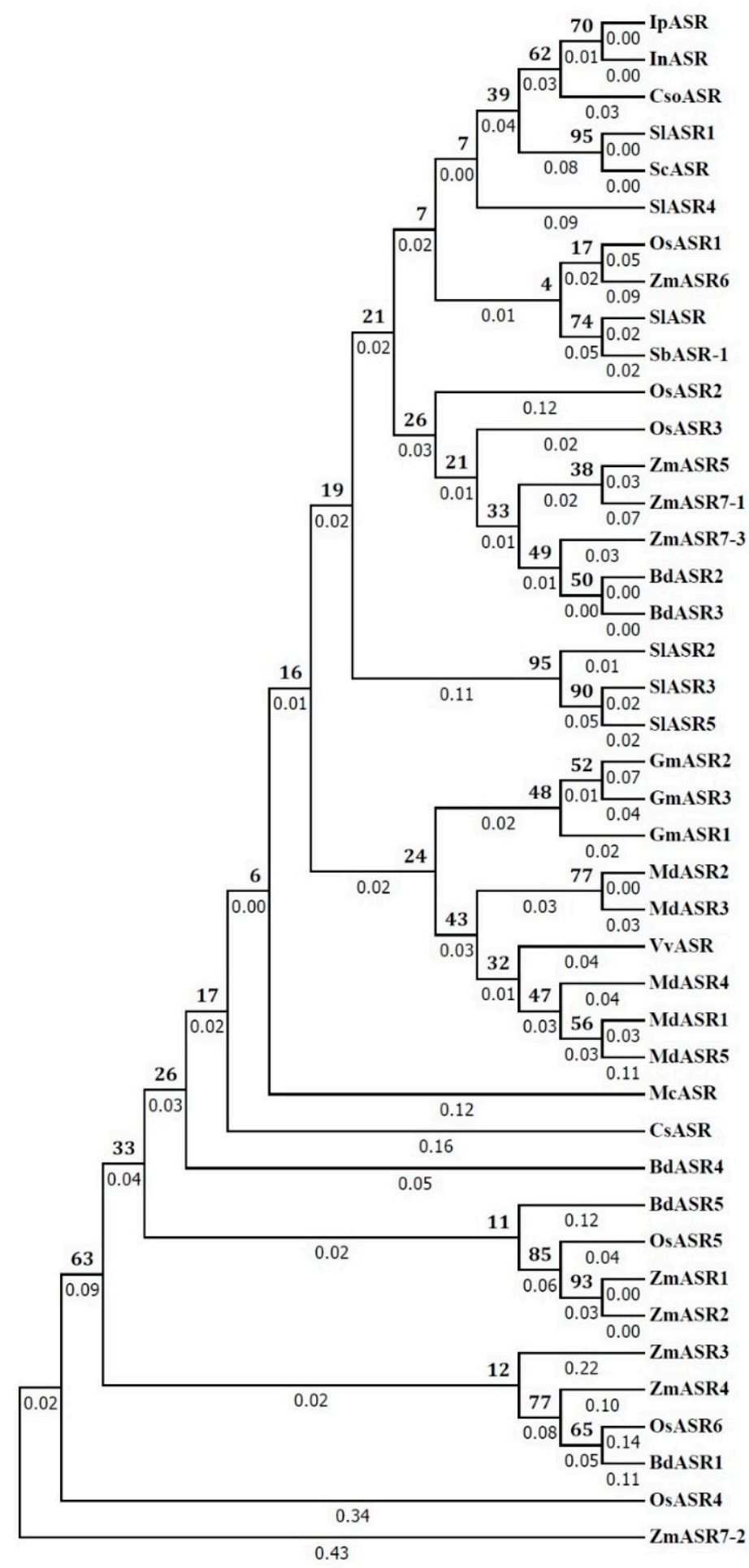

Figure 2. Phylogenetic analysis of IpASR with other ASR proteins. GenBank accession numbers are listed in the "Materials and Methods" (Section 4).

\subsection{Expression Profiles of IpASR}

To investigate the expression pattern of IpASR in I. pes-caprae, qRT-PCR was performed with total RNA extracted from the various tissues. Our results demonstrated that $I p A S R$ was expressed constitutively in most of the I. pes-caprae tissues (Figure 3A). The highest level of IpASR transcription 
was detected in the young roots and young leaves, and the flower petals also showed a high expression of IpASR, while IpASR was weakly expressed in the adult plants tissues/cells.

To determine the expression of IpASR under various stresses, the mRNA of IpASR in the root, vine, and leaf under various stress treatments was also investigated (Figure 3B-F). After challenging with $300 \mathrm{mM} \mathrm{NaCl}$ and $300 \mathrm{mM}$ mannitol, the transcription level of IpASR exhibited a slight increase in the root, vine, and leaf tissues at $2 \mathrm{~h}$, and then decreased at $24 \mathrm{~h}$. Low temperature $\left(0^{\circ} \mathrm{C}\right)$ stress also rapidly and slightly induced the expression of IpASR, peaking at $2 \mathrm{~h}$. Additionally, we also evaluated the expression change of IPASR under MV (methyl viologen) and ABA treatment. Our results showed that both MV and ABA could greatly and rapidly increase the transcription level of IpASR (Figure 3). The expression pattern of IpASR indicated that the biological role of IpASR was involved in plant cellular growth or development, as well as the abiotic stress response.

A

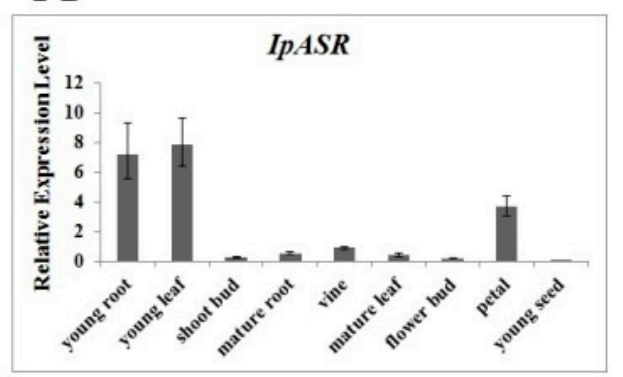

$\mathrm{C}$

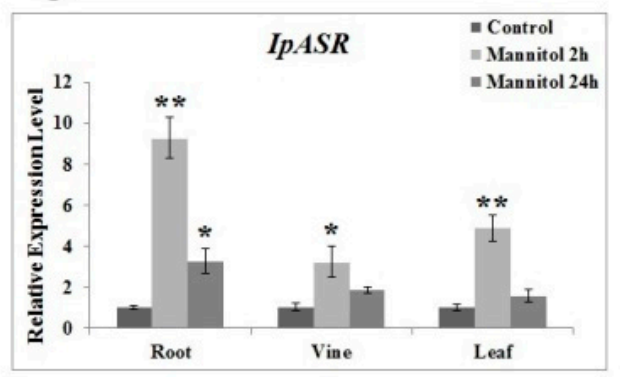

$\mathrm{E}$

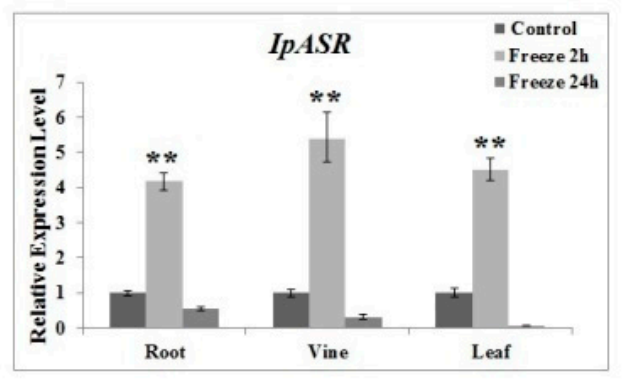

B

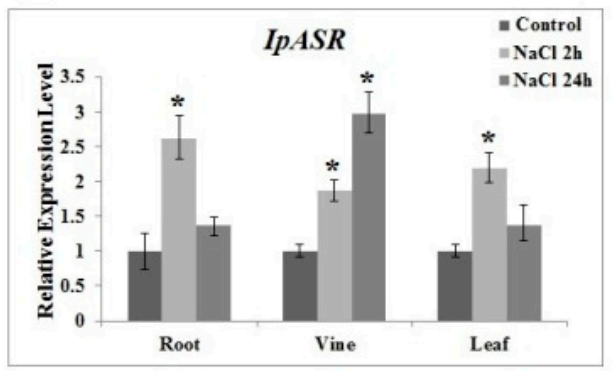

$\mathrm{D}$

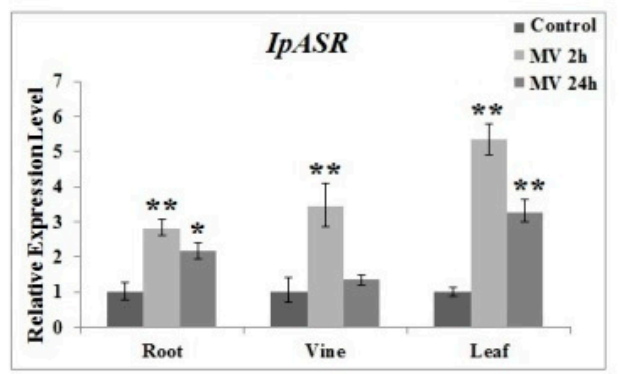

$\mathrm{F}$

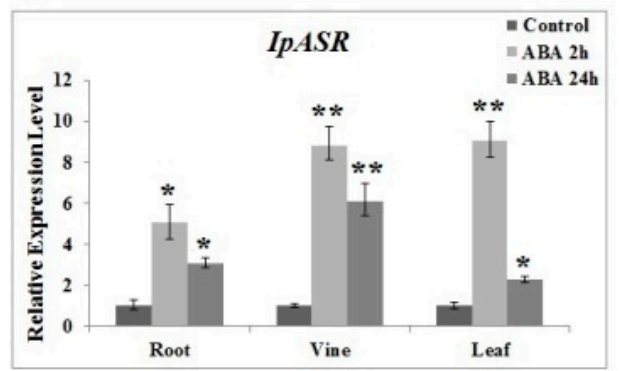

Figure 3. Expression profiles of the IpASR gene among the I. pes-caprae tissues. (A) Differential expression of IpASR in various tissues (young root, young leaf, shoot bud, mature root, vine, mature leaf, flower bud, petal, and young seed). Time-course expression patterns of I $A S R$ in response to different abiotic stresses: $\mathrm{NaCl}$ (B) mannitol simulating dehydration (C) MV (methyl viologen), (D) freezing, (E) and abscisic acid (ABA) treatment. (F) Values are the means \pm standard deviation (SD) $(n=3)$. Asterisks indicate significant differences from the control (Student's $t$-test $p$ values, ${ }^{*} p<0.05$ and $* * 00.01)$. 


\subsection{IpASR Improves the Stress Tolerance of E. coli}

The ORF sequence of the IpASR cDNA was subcloned into the pGEX 6p-1 vector and GST-IpASR was expressed in E. coli BL21 (DE3) cells. High accumulation of GST-IpASR and GST (control) proteins were observed at $4 \mathrm{~h}$ after inducing with isopropyl $\beta$-D-thiogalactopyranoside (IPTG). The differences in molecular weights between the fusion GST-IpASR and GST proteins confirmed the predicted 24-kDa molecular weight of the IpASR protein (Figure 4A).

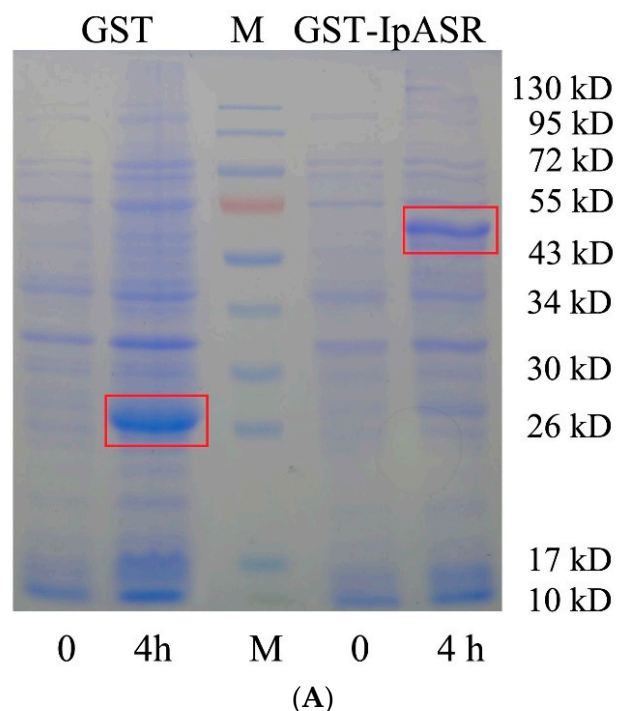

Salinity stress
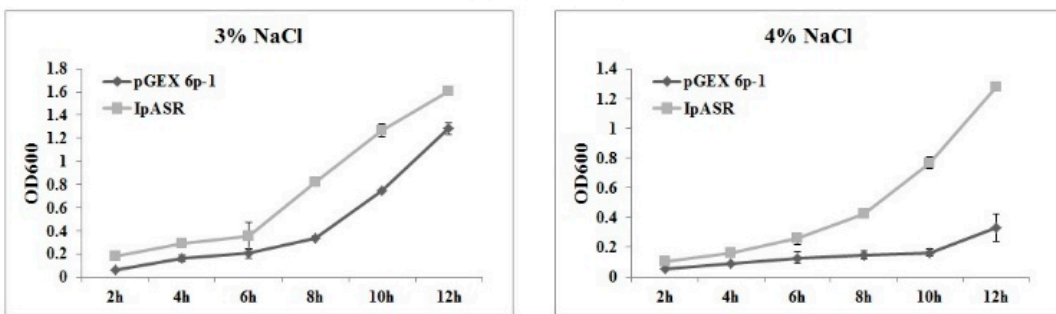

Osmotic stress
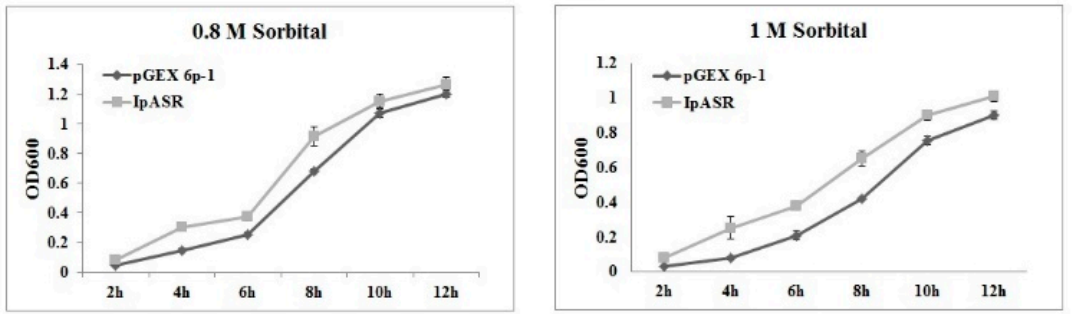

Oxidative stress
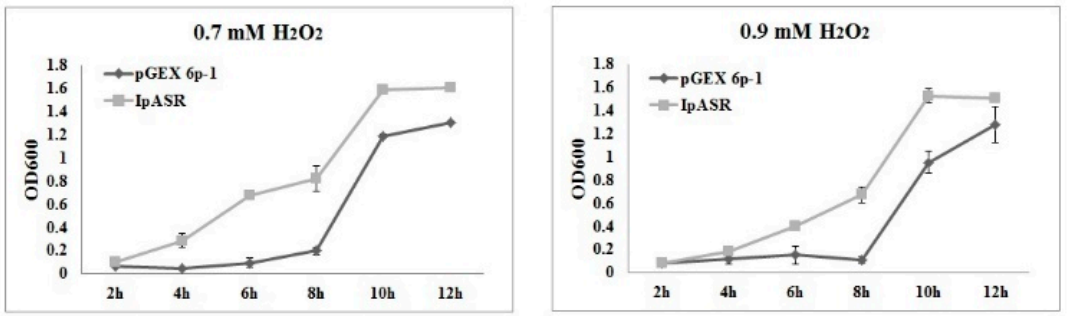

(B)

Figure 4. Cont. 


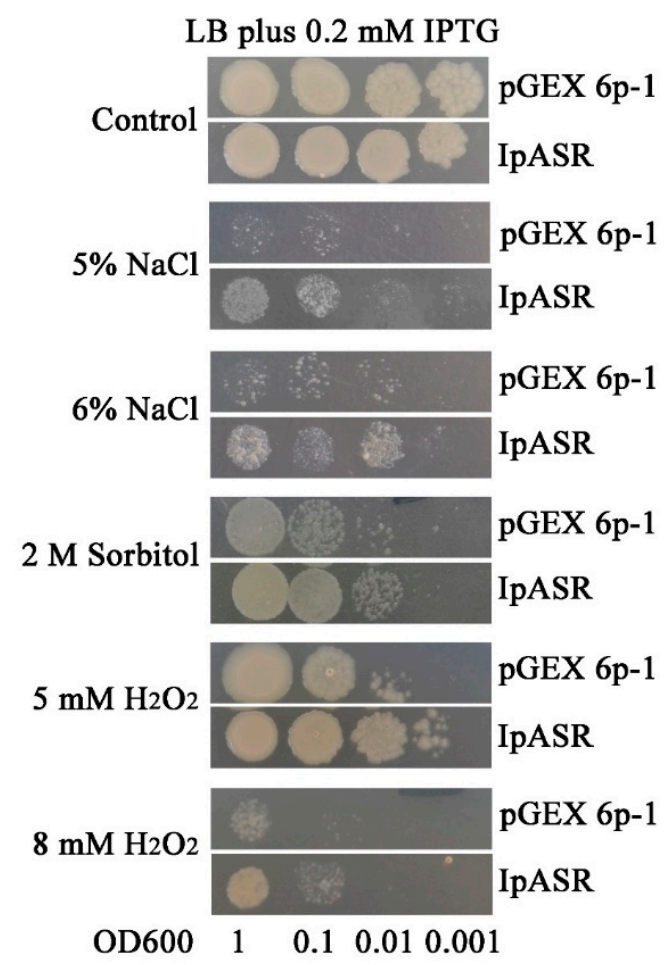

(C)

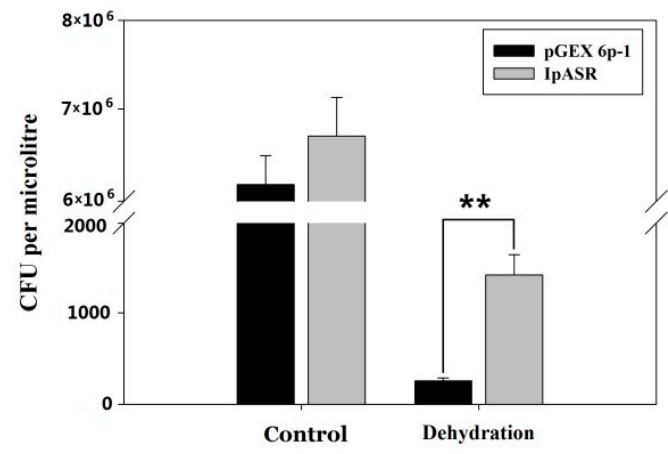

(D)

Figure 4. Functional analysis of IpASR induced-expression for salt, drought, and $\mathrm{H}_{2} \mathrm{O}_{2}$ tolerance in E. coli. (A) Induced expression of the GST-IpASR (IpASR-pGEX 6p-1) fusion protein and single GST (pGEX 6p-1 empty vector) protein in E. coli. 0 and $4 \mathrm{~h}$ : The respective IPTG (isopropyl $\beta$-D-thiogalactopyranoside) induction times. (B) The growth performance of E. coli BL21 (pGEX 6p-1, upper)/(IpASR-pGEX 6p-1) on Luria-Bertani (LB) plates containing stress factors. Control (top): LB medium; $5 \% \mathrm{NaCl}$ : LB medium containing $5 \% \mathrm{NaCl} ; 6 \% \mathrm{NaCl}$ : LB medium containing $6 \% \mathrm{NaCl}$; $2 \mathrm{M}$ Sorbitol: LB medium containing $2 \mathrm{M}$ sorbitol; $5 \mathrm{mM} \mathrm{H}_{2} \mathrm{O}_{2}$ : LB medium containing $5 \mathrm{mM} \mathrm{H}_{2} \mathrm{O}_{2}$; $8 \mathrm{mM} \mathrm{H}_{2} \mathrm{O}_{2}$ : LB medium containing $8 \mathrm{mM} \mathrm{H}_{2} \mathrm{O}_{2}$. The cell cultures were adjusted to $\mathrm{OD}_{600}$ until 1.0 and then diluted serially $(1: 10,1: 100$, and 1:1000, respectively). Two microliters of each sample was spotted onto the LB plates containing 0.2 mM IPTG. (C) Growth kinetics of E. coli transformed with pGEX 6p-1 (control) and IpASR-pGEX 6p-1. Cells were grown until the OD at $600 \mathrm{~nm}$ reached 0.5 , following which $0.2 \mathrm{mM}$ IPTG was added, and the cells were incubated for $4 \mathrm{~h}$ at $30^{\circ} \mathrm{C}$. The cells were then transferred into fresh LB medium (1:100, plus $0.2 \mathrm{mM}$ IPTG) supplied with different concentrations of $\mathrm{NaCl}(3 \%$ or $4 \%$ ), sorbitol $(0.8$ or $1 \mathrm{M})$, or $\mathrm{H}_{2} \mathrm{O}_{2}(0.7$ or $0.9 \mathrm{mM})$. The bacteria were cultured at $37^{\circ} \mathrm{C}$ and $200 \mathrm{rpm}$. The $\mathrm{OD}_{600}$ values were measured every $2 \mathrm{~h}$ to evaluate the growth conditions. (D) Cell viability relates to $\mathrm{CFU}$ (colony former unit) before (control) and after desiccation $\left(40^{\circ} \mathrm{C}\right.$ for $4 \mathrm{~h}$ ). Error bars indicate the SD based on three replicates. Asterisks indicate significant differences from the control (Student's $t$-test $p$ values, ${ }^{* *} p<0.01$. 
The effect of IpASR accumulation on E. coli stress tolerance was assessed by spot and liquid culture assays. When assayed with the liquid culture method under stress conditions, the growth of $E$. coli cells expressing the GST-IpASR fusion protein increased significantly with time compared to the BL21-DE cells expressing GST only (Figure 4A). Cells harboring GST-IpASR demonstrated better performance and a quicker growth rate against $\mathrm{NaCl}(3 \%$ and $4 \%)$, sorbitol $(0.8$ and $1 \mathrm{M})$, and $\mathrm{H}_{2} \mathrm{O}_{2}$ (0.7 and $0.9 \mathrm{mM}$ ) stresses compared to the control E. coli BL21 (DE3) cells expressing GST only (Figure 4B). Comparable E. coli stress tolerances were observed for the spot assay, where the number of colonies was higher in the stress treatments for recombinant cells compared to E. coli and the vector (GST) control (Figure 4C).

The tolerance of E. coli with the empty vector (GST) control and GST-IpASR subjected to drought stress was also determined by spreading the culture dilutions on Luria-Bertani (LB) plates containing $0.2 \mathrm{mM}$ IPTG after drying the bacterial precipitate at $40{ }^{\circ} \mathrm{C}$ for $4 \mathrm{~h}$, and recovering it in $100 \mu \mathrm{L}$ liquid LB medium for $1 \mathrm{~h}$. Figure 4D shows a clear difference in the number of viable $E$. coli cells before and after desiccation stress. Before the drying process, very similar CFUs (colony former unit, expressed in $\times 10^{6}$ units) were obtained for the control (GST) and GST-IpASR-expressing cells, whereas after desiccation, although only a very small fraction of the cells survived, the number of CFUs expressing GST-IpASR was five times higher than in the control cells. This result suggests that IpASR expression in E. coli improved its survival capacity after desiccation.

\subsection{IpASR Localizes to the Cell Nucleus}

To investigate the subcellular localization of IpASR, a transient expression assay was performed with the IpASR-GFP fusion protein and GFP (green fluorescent protein) alone (as a control) in Arabidopsis mesophyll protoplasts. The fluorescence of IpASR-GFP was mainly distributed in the nucleus, with similar distribution patterns as NLS-mCherry, while the GFP was distributed throughout the protoplast cells (Figure 5). The results obtained in the protoplasts, in combination with the protein sequence analyses of IpASR (Figure 1), indicated that IpASR localized to the cell nucleus as a nuclear protein.

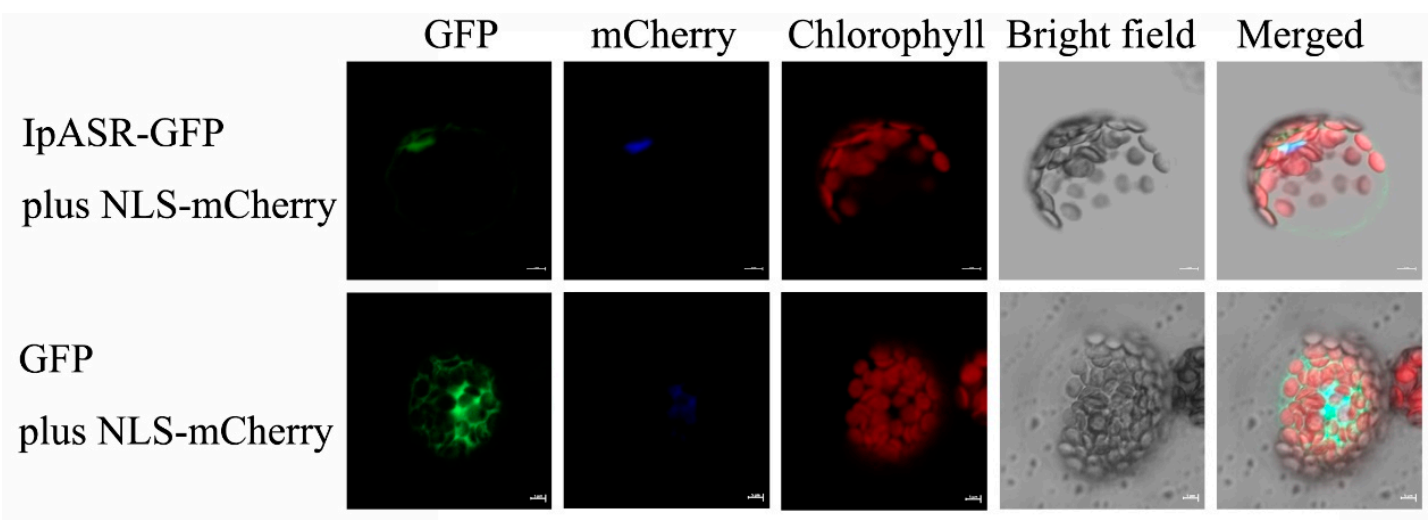

Figure 5. Subcellular localization of IpASR. Arabidopsis protoplasts expressing the IpASR-GFP fusion protein (upper) and GFP (lower) observed under a laser scanning confocal microscope. The blue color indicates the nucleus using mCherry as the nuclear marker. The red color indicates the autofluorescence emitted by chloroplasts. The bar represents $5 \mu \mathrm{m}$.

\subsection{IpASR Exhibits Transcriptional Activity}

ASRs from other plants species have been reported to possess transactivation activity $[4,9]$. To assess the transactivation activity of IpASR, its complete coding region was fused in-frame with the GAL4 DNA binding domain of the pGBKT7 vector in this study. Furthermore, three truncated IpASR fragments were also generated into the pGBKT7 vector with the purpose of categorizing the crucial 
region of IpASR for driving transcription (Figure 6A). The yeast growth on the SD medium lacking tryptophan, leucine, and histidine (SD/-Trp/-Leu/-His), and the staining assay of $\alpha$-galactosidase activity in Figure 6B, indicate that the transactivation sites of IpASR are located in the N-terminal of IpASR.

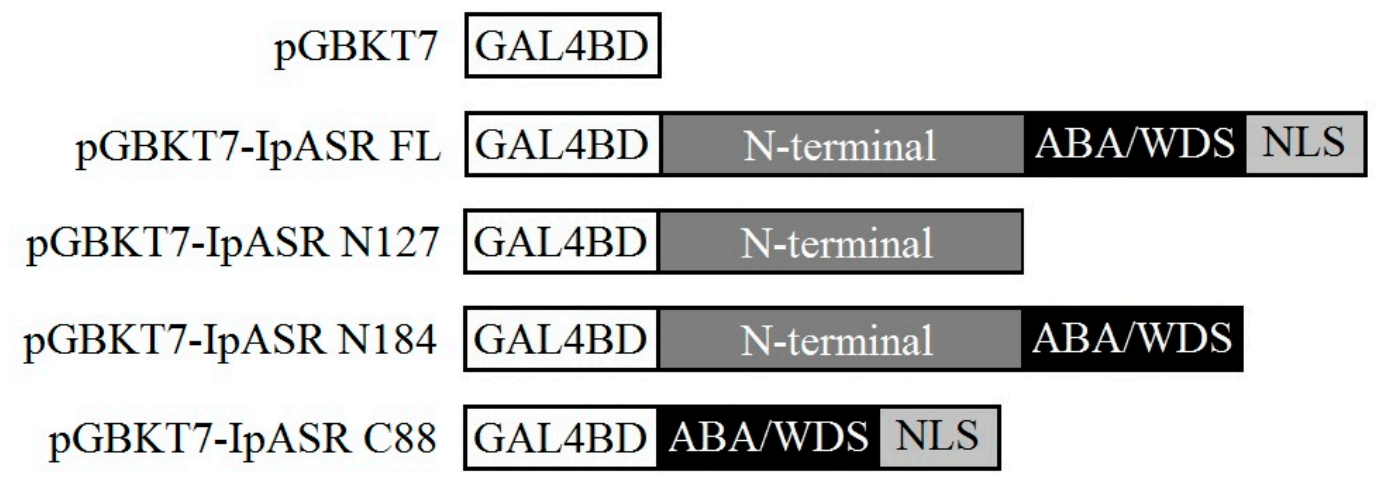

(A)

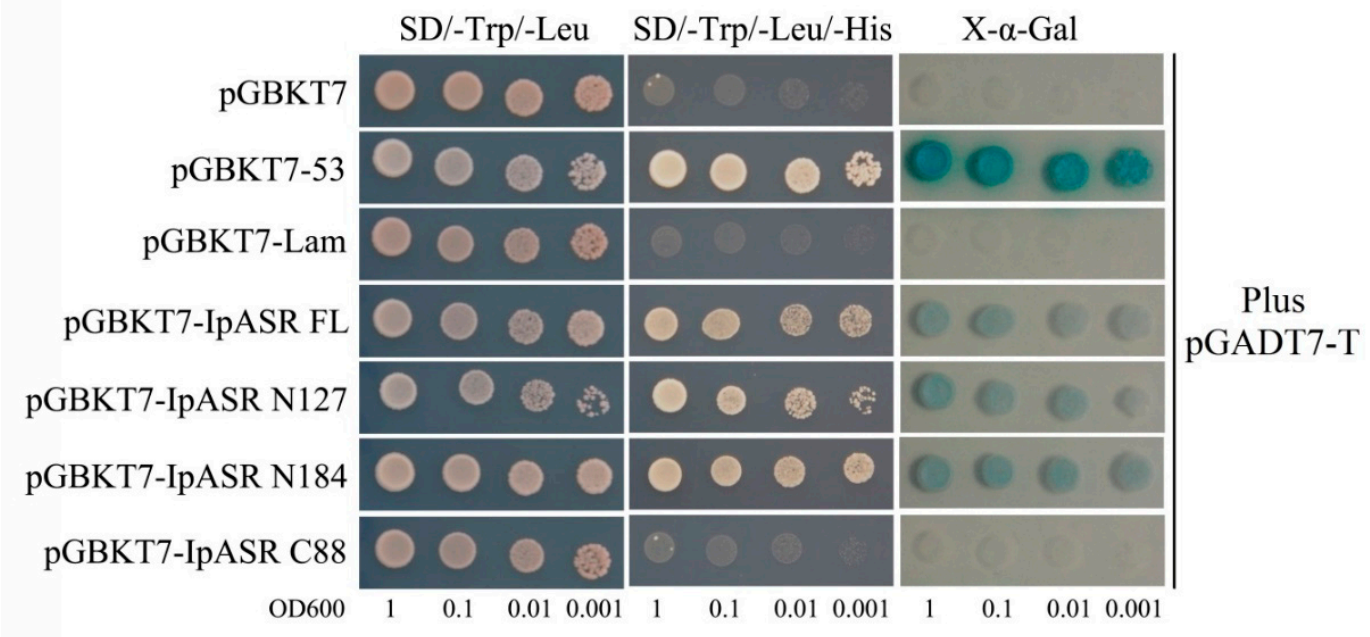

(B)

Figure 6. GAL4 DNA binding domain-IpASR fusion analyses for transactivation activity and $\alpha$-galactosidase assay in yeast. (A) The GAL4 DNA binding domain was fused with different parts of IpASR and transformed into the yeast strain AH109 containing the His3 and MEL1 reporter genes. (B) Analysis of $\alpha$-galactosidase activity of the relative yeast strains on plates. The yeast culture $\left(\mathrm{OD}_{600}\right.$ to 1) was serially diluted to $\mathrm{OD}_{600}$ values of $0.1,0.01$ and 0.001 , and then the 2- $\mu \mathrm{L}$ yeast liquid was spotted onto SD plates and cultured for 2 days at $30^{\circ} \mathrm{C}$.

\subsection{IpASR Improves Abiotic Stress Tolerance in Transgenic Arabidopsis}

To assess the function of IpASR in stress tolerance, transgenic Arabidopsis plants overexpressing $I p A S R$ under the control of the CaMV35S promoter were generated. Finally, two independent homozygous IpASR T3 lines (IpASR OX3 and IpASR OX8) with high expression levels were selected for further functional analysis (Figure 7). 
A
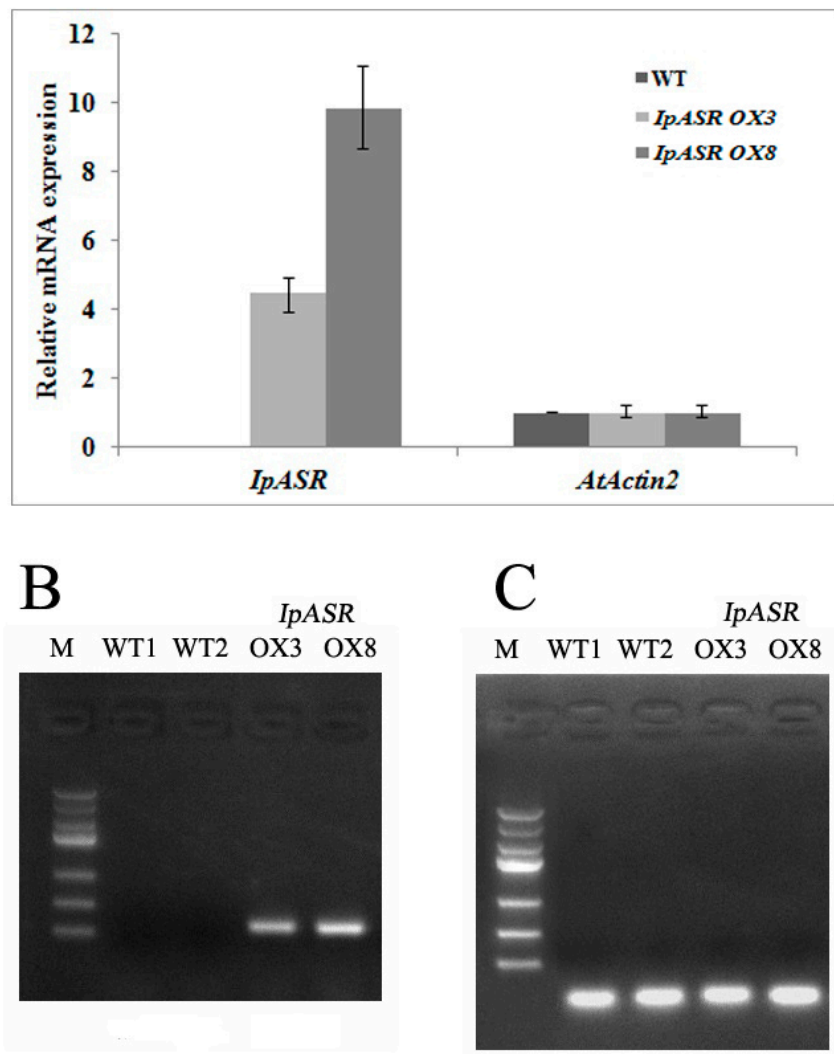

Figure 7. Overexpression analyses of IpASR in transgenic Arabidopsis lines (IpASR OX3 and IpASR OX8). (A) Quantitative RT-PCR analysis of IpASR in transgenic Arabidopsis lines and wild type (WT) Arabidopsis. Actin 2 was used as an internal control. Error bars indicate the SD based on three replicates. (B) RT-PCR analysis of IpASR in transgenic Arabidopsis lines and two WT lines. (C) RT-PCR analysis of AtACT2 in transgenic Arabidopsis lines and two WT lines as a control.

Under normal conditions (Murashige and Skoog medium, MS medium), there were no obvious differences in seed germination and seedling growth between transgenic and wild type (WT) Arabidopsis. However, the IpASR OX plants grew better than the WT plants on MS medium supplemented with $200 \mathrm{mM}$ mannitol or $100 \mathrm{mM} \mathrm{NaCl}$ (Figure 8A). They displayed larger cotyledons than the WT plants, and the seed germination rates of the transgenic lines were significantly greater than those of the WT plants, and corresponded with the increased mannitol (200, 300 and $400 \mathrm{mM})$ and salinity $(150,175$ and $200 \mathrm{mM}$ ) concentrations (Figure 8B,C). When grown on MS medium containing different concentrations of mannitol $(200$ and $300 \mathrm{mM})$ or $\mathrm{NaCl}(150 \mathrm{mM})$ for three weeks, the WT and IpASR OX line seedlings exhibited an obvious difference (Figure 9). These results indicated that IpASR could enhance salt and osmotic stresses tolerance in transgenic Arabidopsis in both the seed germination and growth stages. 
A

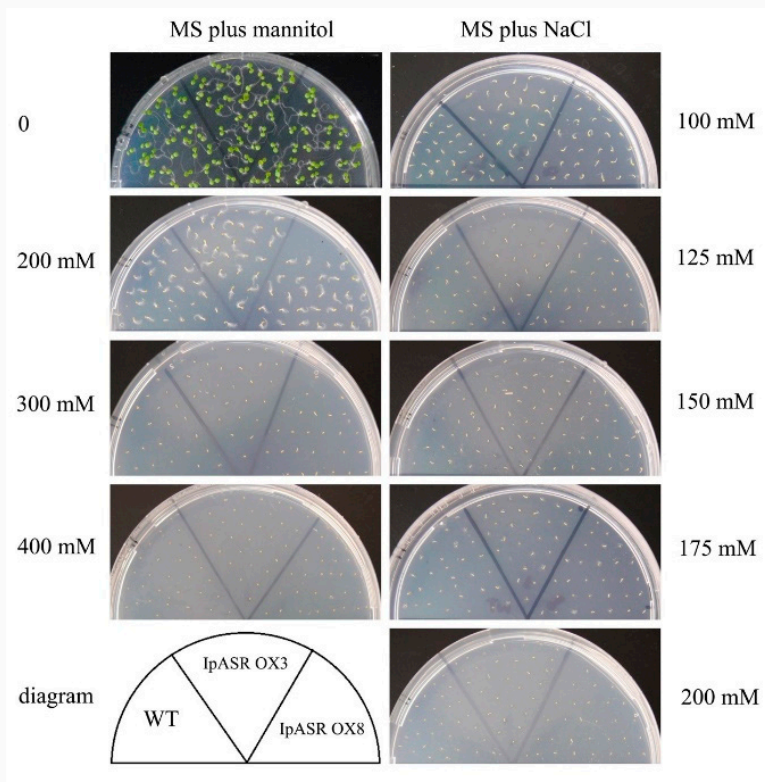

B

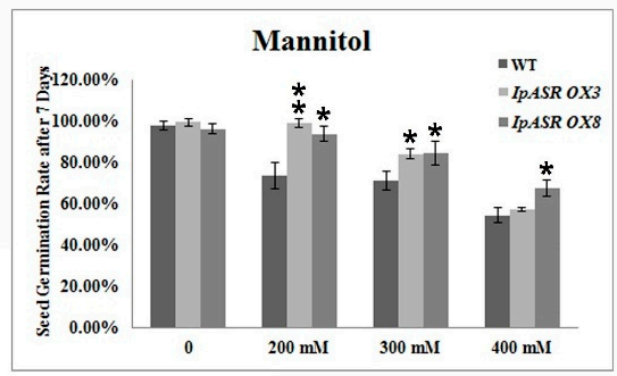

C

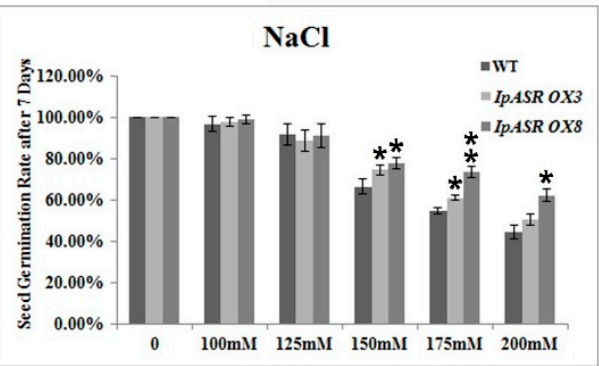

Figure 8. Osmotic and salt stress analyses of transgenic plants with $I p A S R$ with regards to seed germination rate. (A) Photographs of transgenic lines (IpASR OX3 and IpASR OX8) and WT seeds germinated on MS (Murashige and Skoog) medium or MS medium with mannitol (left, 200, 300 and $400 \mathrm{mM}$ ) or $\mathrm{NaCl}$ (right, 100, 125, 150, 175 and $200 \mathrm{mM}$ ) for 7 days. (B,C) Seed germination rates in WT and transgenic lines under mannitol (B) and $\mathrm{NaCl}(\mathbf{C})$ stress after 7 days. Error bars indicate the $\mathrm{SD}$ based on over three replicates $(n \geq 3)$. Asterisks indicate significant differences from the control (Student's $t$-test $p$ values, ${ }^{*} p<0.05$ and ${ }^{* *} p<0.01$ ).

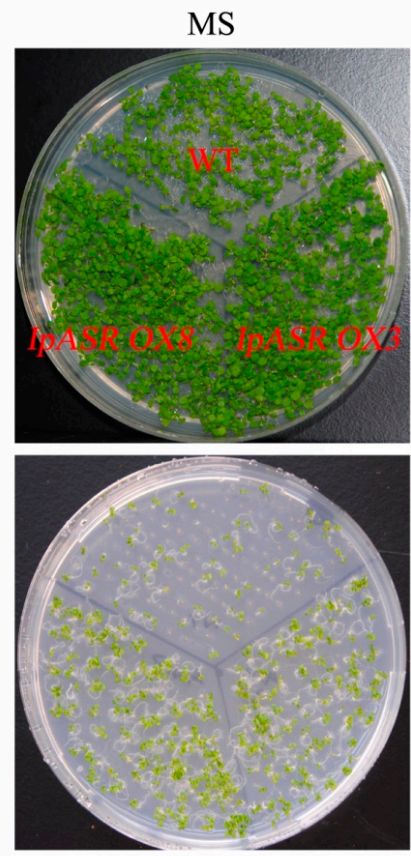

MS plus $200 \mathrm{mM}$ mannitol

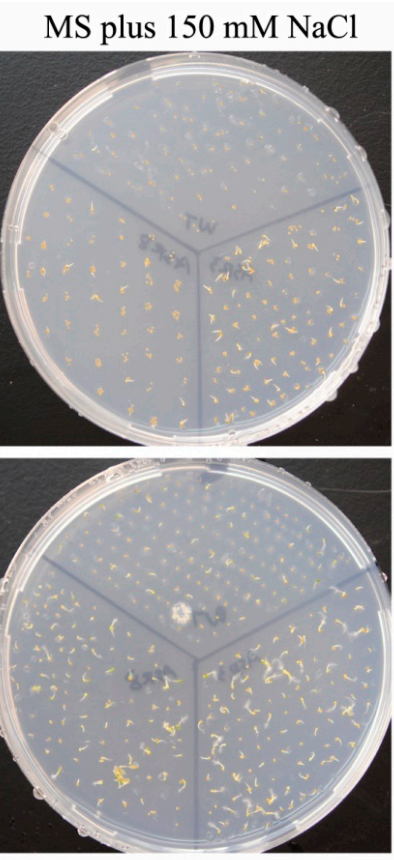

MS plus $300 \mathrm{mM}$ mannitol

Figure 9. Photographs of transgenic lines (IpASR OX3 and IpASR OX8) and WT seeds germinated on MS medium or MS medium with mannitol (200 and $300 \mathrm{mM})$ or $\mathrm{NaCl}(150 \mathrm{mM})$ for 3 weeks. 
To further test the salt and osmotic tolerance, 4-day-old seedlings were transferred to MS agar medium containing $\mathrm{NaCl}(100,125$ and $150 \mathrm{mM})$ or mannitol (200, 300 and $400 \mathrm{mM})$ and further cultivated for 7 days, following which the root lengths were measured. When grown on medium without $\mathrm{NaCl}$ or mannitol, no significant differences were observed between the WT and transgenic plants (IpASR OX3 and IpASR OX8). In contrast, the root lengths of both sets of transgenic plants were significantly longer than the WT plants when grown on medium containing $\mathrm{NaCl}$ or mannitol, suggesting that the overexpression of IpASR enhances salt and osmotic tolerance at the seedling stage (Figure 10).
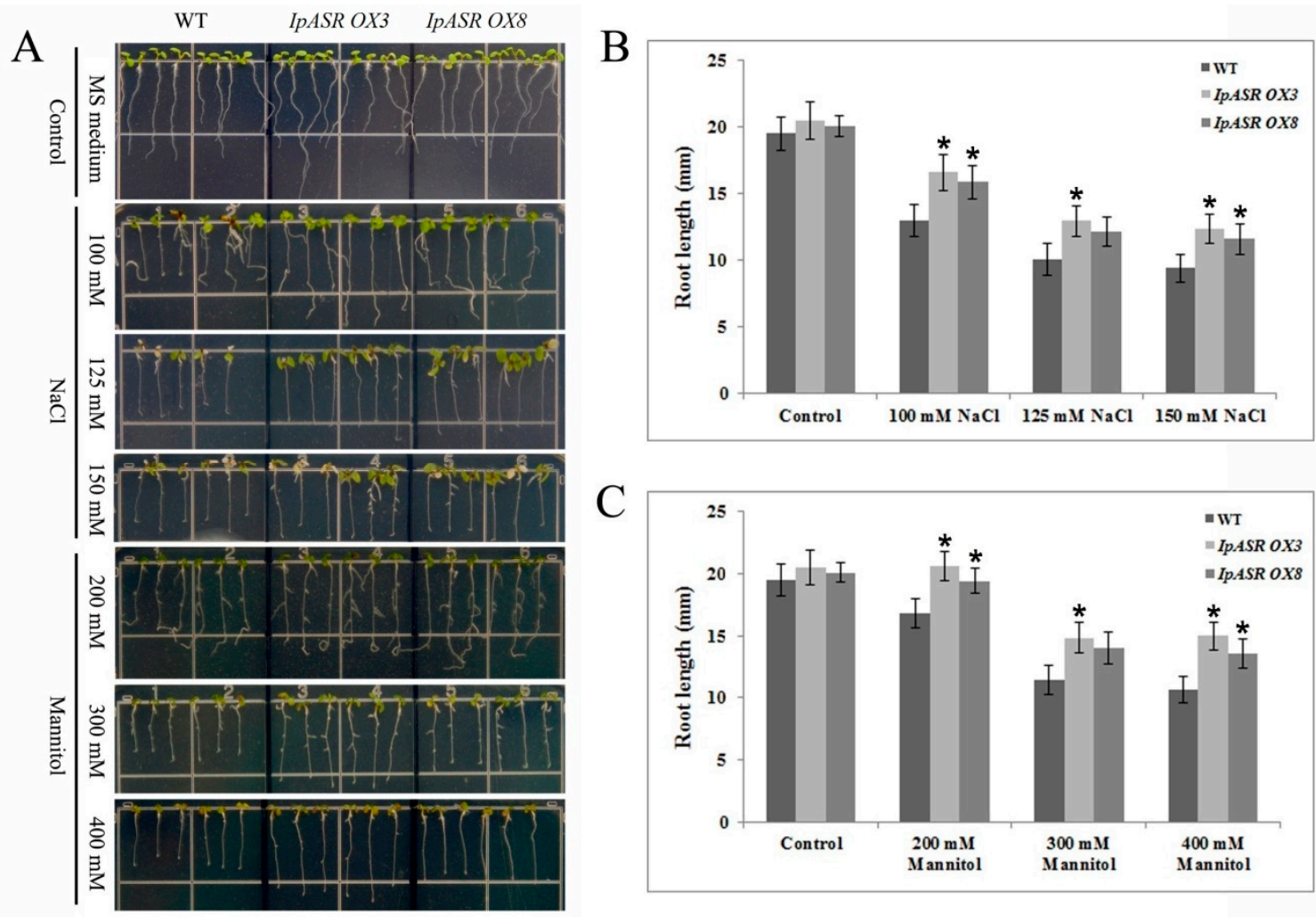

Figure 10. Osmotic and salt stress analyses of transgenic plants with IpASR based on seedling root length. Four-day-old seedlings were transplanted into MS medium containing $\mathrm{NaCl}$ or mannitol and then grown for $7 \mathrm{~d}$ before measuring the root length. (A) Photographs of transgenic lines (IpASR OX3 and IpASR OX8) and WT seedlings on MS medium or MS medium with $\mathrm{NaCl}(100,125$ and $150 \mathrm{mM})$ or mannitol (200, 300 and $400 \mathrm{mM})$; (B,C) Seedling root length $(\mathrm{mm})$ in WT and transgenic lines under $\mathrm{NaCl}(\mathbf{B})$ and mannitol (C) stress after 7 days. Error bars indicate the SD based on over three replicates $(n \geq 3)$. Asterisks indicate significant differences from the control (Student's $t$-test $p$ values, ${ }^{*} p<0.05$.

To further assess the function of IpASR during salt and drought tolerance, the corresponding treatments were applied to 3-week-old transgenic and WT plants grown under normal conditions with watering withheld for 10 days. The plants were then subjected to salt or drought treatment. At salinity stress levels of 150 and $200 \mathrm{mM} \mathrm{NaCl}$, almost all the WT plants showed severely reduced growth, and even death, while the IpASR OX lines showed slight chlorosis and generally exhibited sustained growth and resistance to salinity stress (Figure 11A,B). Under normal conditions, there were no differences in the growth of the transgenic lines and the WT controls.

Additionally, the IPASR transgenic plants also showed characteristics of drought tolerance. The adult Arabidopsis plants (including IpASR OX3, IpASR OX8, and WT) for which watering was withheld for 10 days were primed for the drought-tolerance assay. After drought treatment, the majority of the WT plants exhibited obvious wilting due to severe dehydration, while the survival 
rates of the transgenic lines (IpASR OX3 and IpASR OX8) were evidently higher than that of the WT controls (Figure 11C). After rewatering, the IpASR OX lines were largely restored, while the WT controls showed a lethal phenotype (Figure 11C).

The above results indicated that IPASR elevated the plant tolerance to salinity and drought stress and significantly improved the survival rates of transgenic Arabidopsis under osmotic or dehydration stress (Figure 11D). Furthermore, these findings indicate that the accumulation of IpASRs in transgenic Arabidopsis provides enhanced protection for basic cellular activities in vivo under osmotic stress.

A

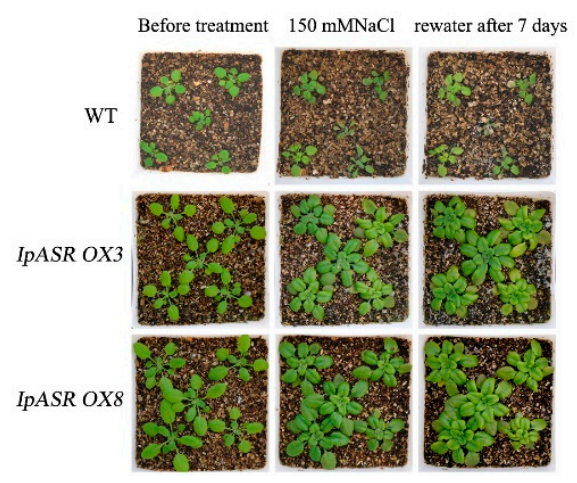

$\mathrm{C}$

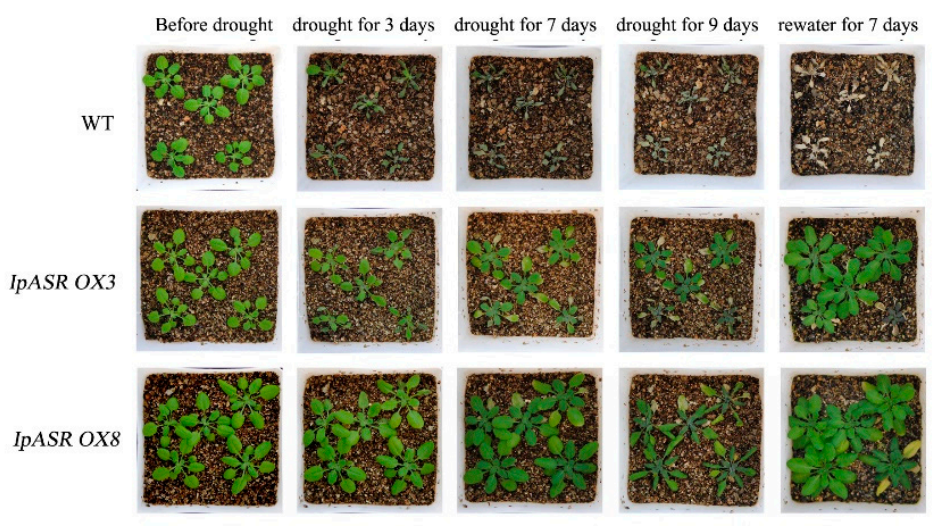

B

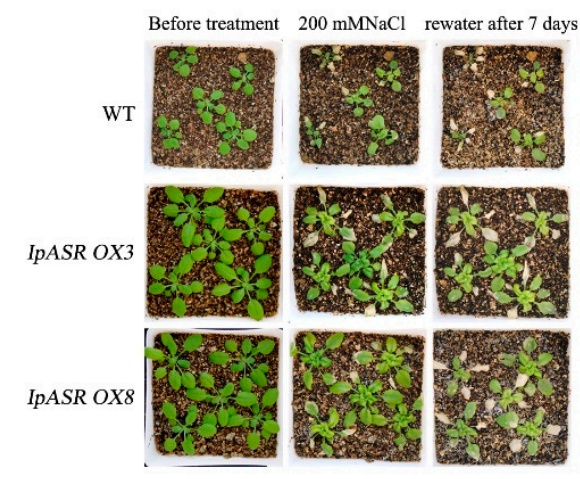

$\mathrm{D}$

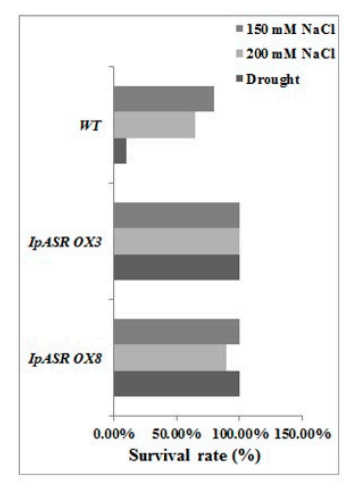

Figure 11. Photographs and survival rates of the transgenic overexpression lines and WT plants grown in pots under normal and salt/drought conditions. (A) The effects of $150 \mathrm{mM} \mathrm{NaCl}$ on transgenic lines and WT; (B) the effects of $200 \mathrm{mM} \mathrm{NaCl}$ on transgenic lines and WT; (C) the effects of withholding water on transgenic lines and WT. (D) The statistics for the survival rate of the transgenic lines and WT Arabidopsis after salt/drought stress. Thirty plants each of WT and two transgenic lines (IpASR OX3 and IpASR OX8) were treated with various concentrations of $\mathrm{NaCl}$ or drought stress.

\subsection{Overexpression of IpASR Increases Relative Water Content (RWC) and Proline Content, and Decreases Ion} Leakage (IL) and Malondialdehyde (MDA) Content under Osmotic Stress

To further clarify the possible physiological mechanisms involved in cellular protection mediated by IpASR, several physiological indices, including RWC, proline content, ion leakage (IL), and MDA content, which are mainly related to cellular osmotic stress tolerance, were tested in the WT and IpASR transgenic Arabidopsis plants under salt and dehydration treatment. Compared to WT Arabidopsis plants, the RWC and proline content were higher in the transgenic lines when they were subjected to salt stress (150 mM NaCl, 1 day) or osmotic stress (300 mM mannitol, 1 day; Figure 12A,B), which indicated that the cells in the transgenic lines demonstrated better water status and stress resistance 
than the WT plants. IL, an important indicator of membrane injury, was higher in the WT plants than in the transgenic lines, suggesting that the transgenic plants suffered less membrane damage than the WT plants (Figure 12C). Accordingly, the MDA levels also displayed a pattern similar to IL, being lower in the transgenic lines relative to WT (Figure 12D). These physiological indices demonstrated that the transgenic lines were more resistant to salt and osmotic stress and indicated the protective roles of IpASR for the cellular membrane system, even for cell vitality under osmotic stress.
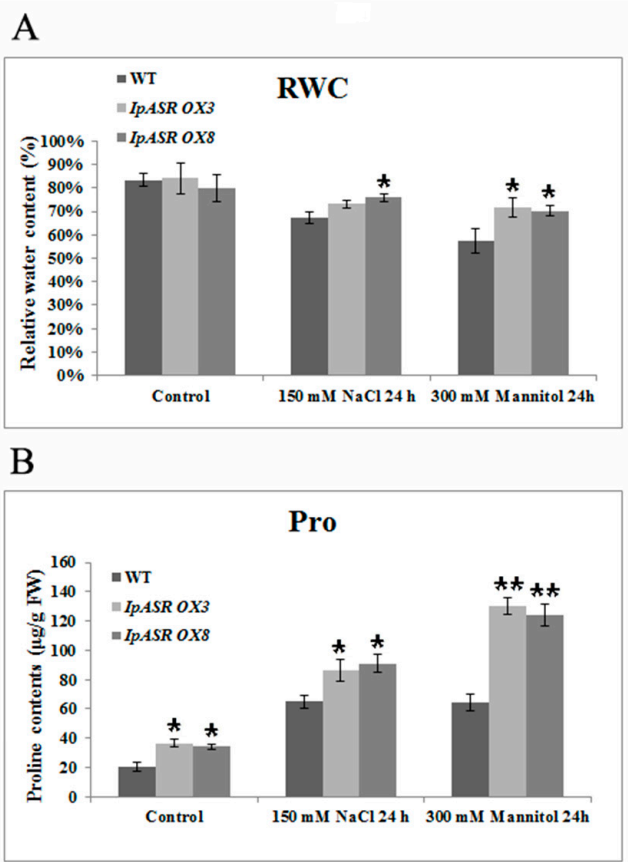

C

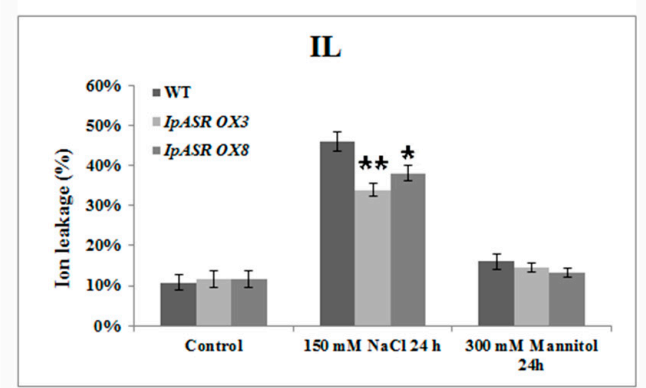

$\mathrm{D}$

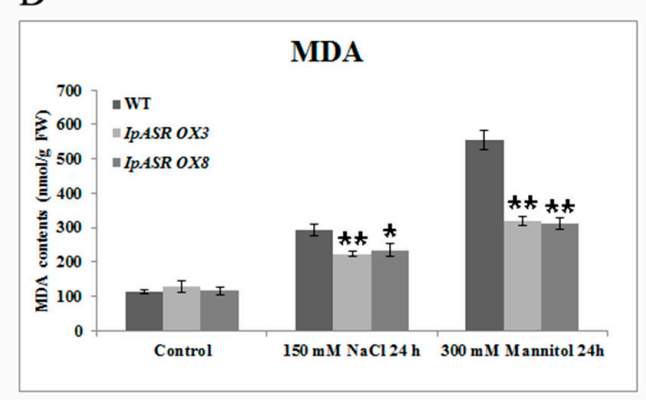

Figure 12. Changes in physiological parameters of IpASR overexpression Arabidopsis and WT seedlings (4 weeks) under $200 \mathrm{mM} \mathrm{NaCl}$ and $200 \mathrm{mM}$ mannitol treatment for $24 \mathrm{~h}$. (A) Relative water content (RWC); (B) Free proline content (Pro); (C) Ion leakage (IL); and (D) Malondialdehyde (MDA). Error bars indicate the SD based on over three replicates $(n \geq 3)$. Asterisks indicate significant differences from the control (Student's $t$-test $p$ values, ${ }^{*} p<0.05$ and ${ }^{* *} p<0.01$ ). 
2.9. IpASR Overexpression Plants Resist Oxidative Damage by Increasing Reactive Oxygen Species ROS Scavenger Accumulation

From Figure 12, it is evident that the MDA and proline contents differed between the WT and IpASR overexpression plants, which suggest that the cells in the IpASR transgenic plants showed stronger activity and better resistance to osmotic stress, including salt and drought. Proline and MDA are also important indices of plant oxidative stress and cell injury in response to stress conditions [20,21]. ROS are continuously generated as a consequence of abiotic stresses, such as drought, cold, salt and heat, causing a reduction in plant growth and crop yield losses worldwide [22]. When plant cells are subjected to stress, proline not only acts as an osmolyte, but also as a ROS scavenger [21], whereas the accumulation of MDA is often the final product of membrane lipid peroxidation caused by ROS accumulation. In other words, the differences in physiological indices between the WT and IpASR transgenic plants are eventually reflected in the cellular ROS accumulation and scavenging caused by osmotic stress. Therefore, it was essential that ROS accumulation be detected in the IpASR transgenic lines and WT. From Figure 13, the $\mathrm{H}_{2} \mathrm{O}_{2}$ and $\mathrm{O}_{2}{ }^{-}$content increased both in the WT and IpASR transgenic lines after salt and mannitol (osmotic) stresses. The DAB (3,3'-diaminobenzidine) and NBT (nitro-blue tetrazolium) staining indicated that the WT plants accumulated more $\mathrm{H}_{2} \mathrm{O}_{2}$ and $\mathrm{O}_{2}{ }^{-}$than the two IpASR OX lines (Figure 13A,B). Some enzymatic antioxidants, such as catalase (CAT) and superoxide dismutase (SOD), are believed to play significant roles in ROS scavenging and the cellular ROS balance. In this study, the activities of two significant antioxidant enzymes, CAT and SOD, were measured in the leaves from potted plants. Under normal growth conditions, the IpASR transgenic lines and WT plants showed some differences in CAT and SOD activities (Figure 13C,D). These results suggested that the overexpression of IpASR reduced ROS accumulation by enhancing SOD and CAT activities under osmotic stress.

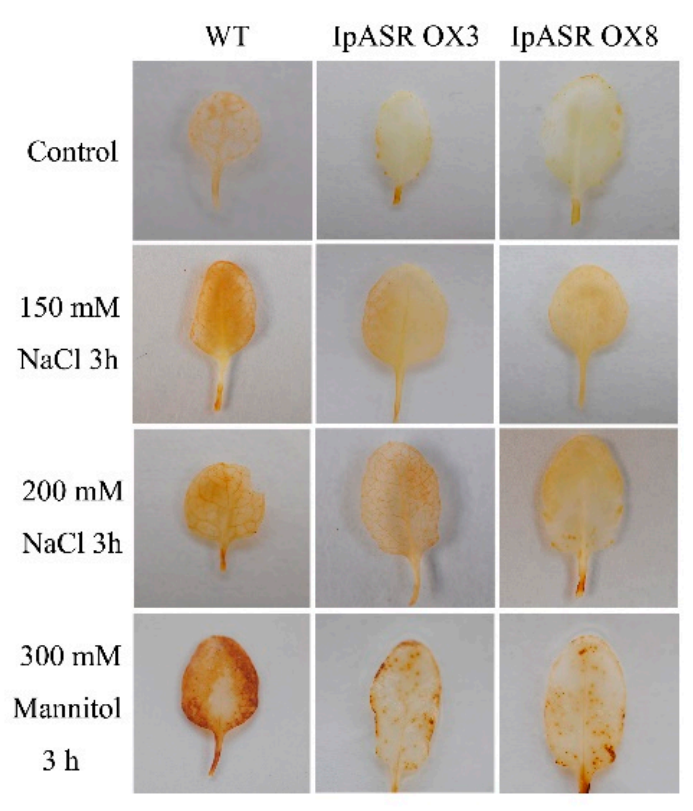

(A)

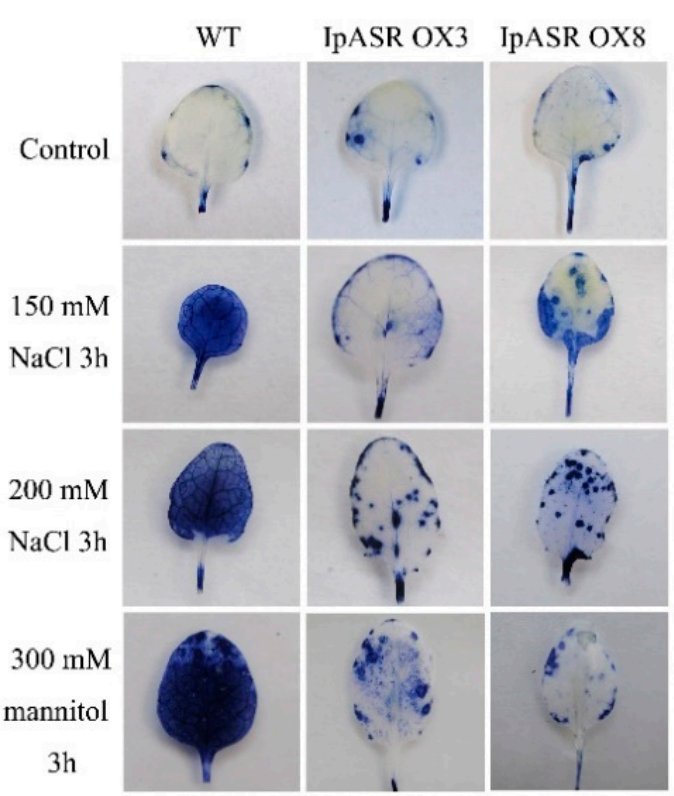

(B)

Figure 13. Cont. 
$\mathrm{C}$

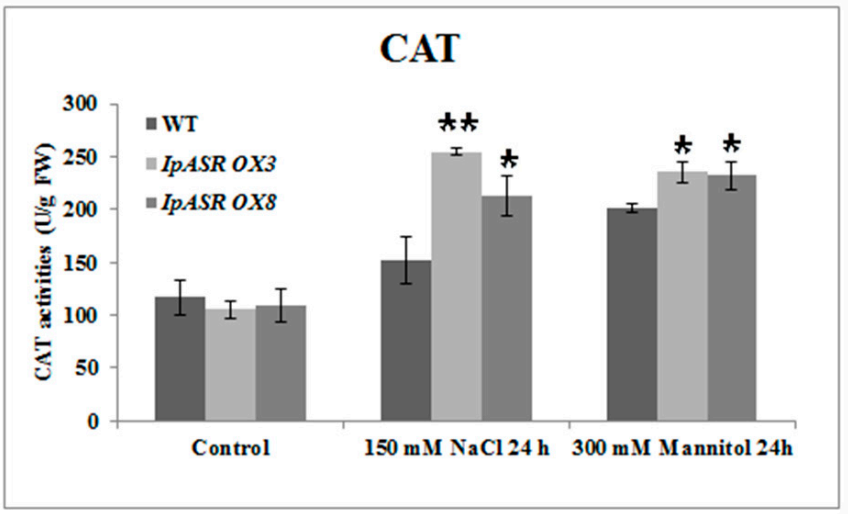

$\mathrm{D}$

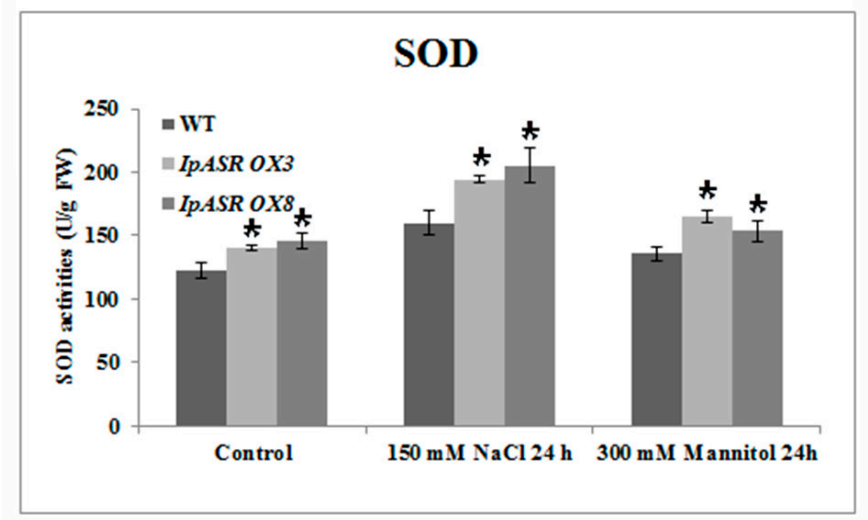

Figure 13. Oxidative stress analyses of transgenic overexpression lines and WT plants. Histochemical staining assays were used to detect $\mathrm{H}_{2} \mathrm{O}_{2}$ and $\mathrm{O}_{2}{ }^{-}$by DAB (A) or nitro-blue tetrazolium (NBT) (B) staining, respectively. (C) Analysis of catalase (CAT) activity in the WT and transgenic lines under normal conditions and osmotic stress; (D) Analysis of superoxide dismutase (SOD) activity in the WT and transgenic lines under normal conditions and osmotic stress. Error bars indicate the SD based on over three replicates $(n \geq 3)$. Asterisks indicate significant differences from the control (Student's $t$-test $p$ values, ${ }^{*} p<0.05$ and $\left.{ }^{* *} p<0.01\right)$.

To examine the possible molecular mechanisms underlying the function of IpASR in salt and dehydration resistance, the expression levels of several oxidation-related genes were analyzed by qRT-PCR in the IpASR transgenic lines and WT plants under $300 \mathrm{mM}$ mannitol and $300 \mathrm{mM} \mathrm{NaCl}$ treatment (Figure 14). The four examined oxidative stress-responsive marker genes (CAT1, CSD1, FSD1 and APX2) in Arabidopsis showed significantly upregulated transcription in the WT and IpASR transgenic plants under stress. This result suggested that IpASR accumulation in plants might upregulate the expression of these oxidative stress-responsive genes, and that these genes might improve plant resistance to salt and osmotic stress. 


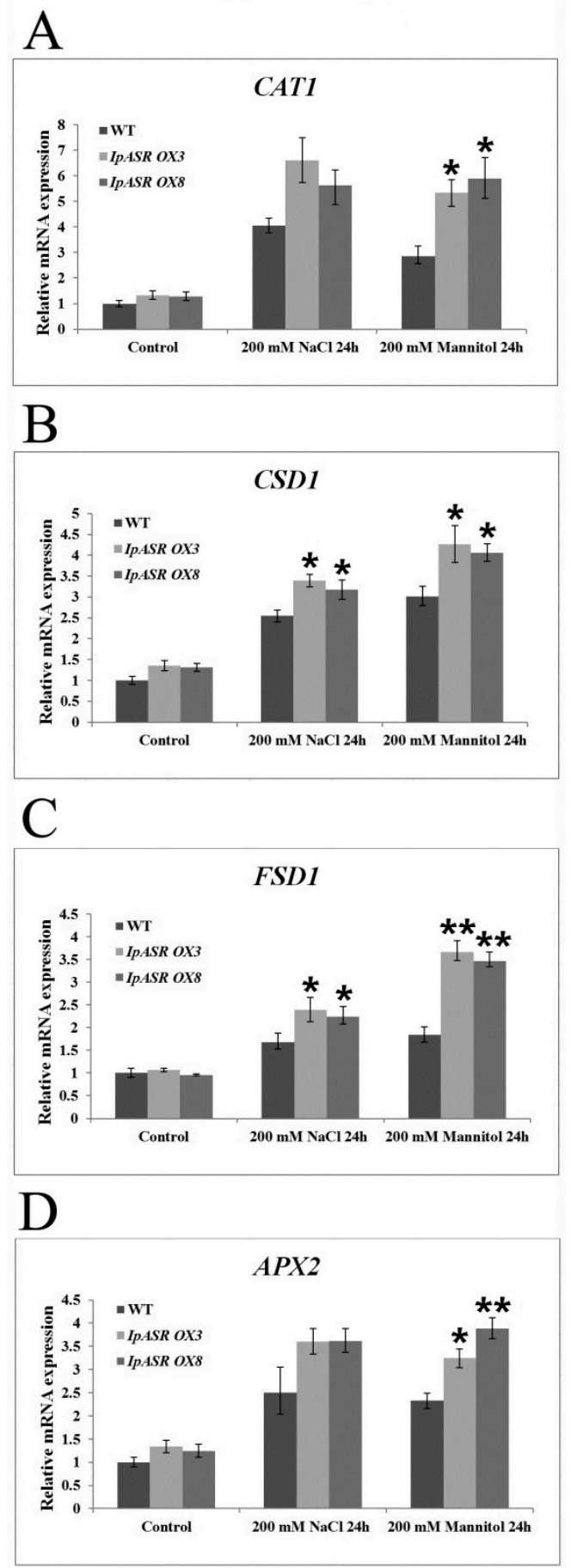

Figure 14. Analysis of the expression levels of reactive oxygen species- (ROS)-related genes in the WT and the transgenic overexpression lines by qRT-PCR under normal and osmotic stress conditions. (A) CAT1; (B) CSD1; (C) FSD1; and (D) APX2. Error bars indicate the SD based on three replicates. Asterisks indicate significant differences from the control (Student's $t$-test $p$ values, ${ }^{*} p<0.05$ and ** $p<0.01)$.

To further confirm the role of IPASR in regulating antioxidant mechanisms, the relationship between IpASR and photo-oxidative stress was also investigated by supplying MV to the IpASR transgenic and WT seedlings. MV is an herbicide that can generate highly reactive, oxygen-centered free radicals within chloroplasts when plants are exposed to sunlight [23]. As shown in Supplementary 
Materials Figure S1, when the plants were supplied with small amount of MV (20 and $50 \mu \mathrm{M}$, spray 3 times per pot $\approx 300 \mu \mathrm{L}$ per pot), after 7 days, there is little differences between WT and IpASR OXs; while after increasing the amount of MV (both 20 and $50 \mu \mathrm{M}$ ) to 12-time spray (about 1.2 mL MV) per pot on the 7th day, after another 7 days, the WT leaves were bleached partly under $20 \mu \mathrm{M}$ MV challenge, and showed almost withered under $50 \mu \mathrm{MMV}$ challenge, while the color of the transgenic lines retained more green under the same concentration (Supplementary Materials Figure S1). Our results indicated that the IpASR transgenic lines demonstrated superior growth status under oxidative stress than the WT plant seedlings.

\section{Discussion}

Till now, only a few plant ASRs have nevertheless been characterized in terms of biological function, with most of them being involved in water deficit stress [4]. When plants suffer from water deficit (such as drought, freezing, high salinity, or ABA signaling caused by other stresses), ASR transcripts are induced, and a large amount of ASR proteins accumulate to reduce the cellular damage caused by the stresses, or to trigger the expression of some other stress response genes and the associated signaling pathways. The exact functions of ASR proteins remain unknown, but have been found to play an important role in improving the adaptability of plants to different abiotic stresses, including water deficit stress [3,24], as well as in regulating plant development and metabolism $[4,25]$.

In the present work, a novel ASR gene, IpASR, was isolated and characterized from the extreme halophyte I. pes-caprae for the first time. Bioinformatics analyses indicated that IpASR is a highly hydrophilic, intrinsically disordered, and highly stable protein, possessing similar characteristics to LEA proteins. The ABA/WDS motif (PF02496) and other characteristics (Table 1) are regarded as key factors in reducing water loss in plants under stress conditions, which is equivalent to the role of hydration buffers in maintaining water balance [26-28]. Combining with the sequence alignment of different ASR members (Figure 1), we can infer that the ABA/WDS motif is highly conserved not only in sequence and secondary structure, but also in biochemical and biological functions, even till now there is no relevant reports about its specific functional mechanisms in plants.

Sequence phylogenetic analysis of IpASR and other plant proteins (Figure 2) showed that IpASR is highly homologous to InASR from morning glory (Ipomoea nil) [29], another Convolvulaceous plant. The expression of InASR in the corolla was upregulated along with flower senescence, while the transcription of IpASR also showed a high level of accumulation in the blooming flower of I. pes-caprae, indicating that it might be involved in the regulation of flower development and senescence as well as dehydration processes. Furthermore, IpASR also showed high homology with another two halophyte members, namely SbASR-1 (ACI15208.1) from S. brachiate [13,14] and SIASR (AGZ20206.1) from S. liaotungensis [15], both of which exhibited a strong positive correlation between transcript accumulation (overexpression assay in tobacco, groundnut, and Arabidopsis) and elevated resistance for salt and drought tolerance. The phylogenetic tree did reveal the close evolutionary relationship between three Convolvulaceous plant ASRs, including IpASR (I. pes-caprae), InASR (I. nil), and CsoASR (C. soldanella). However, due to the phylogenetic tree of ASR proteins was constructed based a few members, the evolutionary relationships of ASRs between glycophyte and halophyte, or monocot and dicot, could not be reflected in this tree. In addition, according to reports by Philippe et al. [30] and Cortés et al. [31], the diversity of the ASR genes was globally low in the context of drought tolerance, and different $A S R$ members undertook different degrees of evolutionary and natural-selection pressure, subsequently presented different haplotype diversity or gene diversity. In summary, the above-mentioned plant ASRs are all involved in water deficit stress. Since the sequence homology usually reflects the functional similarities of different proteins, our bioinformatics analysis revealed that IpASR could be involved in the adaptation of I. pes-caprae to extreme saline and arid environments in tropical and subtropical coastal regions.

Salt, drought, and other abiotic stress factors ultimately result in the significant accumulation of ROS, thereby evoking the antioxidant system of plants [22]. Although there is a lot of evidence 
regarding the participation of plant ASRs in the response to abiotic stress, there is some direct evidence suggesting that plant ASRs have roles in the antioxidant activity of plants [4]. For instance, the overexpression of wheat TaASR1 in tobacco enhances the expression of ROS-related and stress-responsive genes under osmotic stress, thereby improving the drought tolerance and MV resistance of tobacco [28]. The rice ASR1 can both scavenge ROS [32] and act as a transcription factor regulating aluminum responsive genes in rice [33]. A soybean ASR protein can bind metal ions and provide cellular antioxidant protection [34]. In our previous research, IpASR could elevate $\mathrm{H}_{2} \mathrm{O}_{2}$ tolerance when expressed in yeast (data not published), whereas in this report the induction of IpASR in E. coli resulted in the transgenic E. coli exhibiting higher tolerance than the control not only to $\mathrm{NaCl}$ and sorbitol, but also to $\mathrm{H}_{2} \mathrm{O}_{2}$. This further implies that the IpASR protein not only improves the resistance of $E$. coli to osmotic stresses via its highly hydrophilic ability, but at the same time also alleviates the toxicity of ROS to E. coli cells in an unknown manner, thus improving the antioxidant ability of E. coli.

Based on its physical and chemical properties, IpASR exhibits a heightened level of hydrophily, mainly mediated by high contents of hydrophilic amino acids, such as glutamic acid (17.67\%), lysine $(11.63 \%)$, and glycine $(11.16 \%)$. Combined with the ABA/WDS domain, this feature ensures the functionality of IpASR in water deficit. Beside that there is still an important motif, designated as a histidine-rich motif (also named zinc-binding domain) at the $\mathrm{N}$-terminal, which probably mediated the metal-binding (including $\mathrm{Zn}^{2+}, \mathrm{Fe}^{3+}, \mathrm{Ni}^{2+}$ and $\mathrm{Cu}^{2+}$ ) and in turn underwent conformational transition from unfolded to folded, then prevented the formation of ROS, or changed the protein's solubility [7,34]. By analyzing the ASR sequences, we discovered that most ASRs at the $\mathrm{N}$-terminal possess the His-rich motif, as does IpASR. Here we can speculate that this conserved His-rich motif might play some roles in cellular antioxidant responses when challenged by environmental stresses. However, there are still some other ASR members, such as two halophyte ASRs, namely SIASR from S. liaotungensis and SbASR-1 from S. brachiate [13-15], which lack this typical motif. This further illustrates that there might be other mechanisms mediating the response of ASRs to ROS scavenging and abiotic stresses.

The transcription patterns of genes closely reflect the biological function of the genes. Our research indicated that the expression of IpASR was induced by high-salt $(300 \mathrm{mM} \mathrm{NaCl})$, osmotic $(300 \mathrm{mM}$ mannitol), oxidative stress (MV), low temperature $\left(0^{\circ} \mathrm{C}\right)$, and ABA treatment, which further indicated that IpASR is an important stress response gene in I. pes-caprae. Additionally, the IpASR protein is located in the nucleus, suggesting that this protein might be a chaperone or transcription factor in the cell nucleus that alleviates the harm of environmental stress to plant cells. We performed a transcriptional activity assay in yeast, which allowed us to speculate whether IpASR exhibits transcription factor activity and if the action domain is located at the N-terminal (Figure 6).

Over the past few decades, several model plants, such as tobacco and Arabidopsis as well as microorganisms, have been used to carry out the functional validation and characterization of genes from plants $[13,15,16,35,36]$. Here, we expressed IpASR in E. coli for the primary functional validation of IpASR in water deficit and anti-oxidation (Figure 4). To further clarify the function of IpASR under abiotic stress conditions, I $I A S R$ was overexpressed in Arabidopsis plants (Figure 7). Phenotypic analyses indicated that IpASR in Arabidopsis considerably enhances salt and osmotic resistance (Figures 8-11) and also alleviates ROS toxicity (Figures 13 and Supplementary Materials Figure S1). ASR proteins play important roles in abiotic stress tolerance in most plants, as observed for SiASR4, TaASR1, OsASR1/5, and ZmASR1 $[16,28,32,33,37]$. Our research indicated that the overexpression of IpASR in Arabidopsis resulted in stronger tolerance to salt/drought in combination with reduced ROS accumulation and the elevation of the cellular antioxidative enzyme system. Here, we presumed that IpASR could act as a protective protein to maintain enzyme activities or to obstruct ROS production by binding to metals. Furthermore, IpASR exhibits transcription activity (Figure 6), which implies that the accumulation of IpASR affects the expression of some genes. We also assessed the transcripts of some antioxidative genes, and their expression patterns all changed both under control and stress treatments (Figure 12). 
This indicated that IpASR might act as a transcription factor and thus regulate gene expression, with surplus IpASR elevating plant abiotic stress tolerance.

In summary, a salt- and drought-related gene, IpASR, from I. pes-caprae was characterized in this research. Our results indicated that IpASR belongs to ABA/water-deficit stress-related category (PF02496, ABA/WDS motif). The expression of IpASR is induced by mannitol, high salinity, MV, cold, and ABA. The overexpression of IpASR in E. coli and Arabidopsis displayed complex expression patterns involved in responses to abiotic stress, primarily salt and drought tolerance. Nonetheless, IpASR acts as a downstream factor in the response of plants to salinity and drought stresses and may be an important candidate gene for the molecular breeding of salt-tolerant plants. Our findings indicated that IpASR acts as a transcription factor, hydrophilic protein, or an ROS scavenger; and has a pleiotropic effect on physiological processes, thereby improving plant tolerance to multiple abiotic stresses. This report should help elucidate the molecular mechanisms of IpASR from I. pes-caprae in salt and drought resistance, and provides valuable information for the development of crops with enhanced abiotic stress tolerance, ultimately aiding breeding programs aimed at improving salt/drought tolerance.

\section{Materials and Methods}

\subsection{Plant Materials, Growth Conditions, and Stress Treatments}

The I. pes-caprae plants were cultivated in the South China Botanical Garden $\left(23^{\circ} 18^{\prime} 75.91^{\prime \prime} \mathrm{N}\right.$, $113^{\circ} 37^{\prime} 02.38^{\prime \prime}$ E) in Guangzhou city. The I. pes-caprae seeds were collected from the seaside of Zhuhai city $\left(22^{\circ} 16^{\prime} 25.37^{\prime \prime} \mathrm{N}, 113^{\circ} 34^{\prime} 18.00^{\prime \prime} \mathrm{E}\right)$, Guangdong province, China. Different parts of I. pes-caprae were frozen in liquid nitrogen and stored at $-80{ }^{\circ} \mathrm{C}$ until RNA isolation.

For I. pes-caprae seedling culture, the seeds were sterilized with 70\% ethanol and then the seed coats were broken with emery paper prior to being placed onto Murashige and Skoog (MS) basal salts distributed into plates with sand and soil, and seedlings grew outdoors from April to November (2016) in the South China Botanical Garden. The seedlings were used for stress treatment assays to assess the expression patterns of IpASR. Subsequently, salt $(300 \mathrm{mM} \mathrm{NaCl})$, simulated drought or osmotic (300 mM mannitol), oxidative ( $0.1 \mathrm{mM}$ methyl viologen), and cold $\left(0{ }^{\circ} \mathrm{C}\right)$ stresses and ABA treatment $(0.1 \mathrm{mM})$ were applied to the I. pes-caprae seedlings to detect the expression patterns of IpASR.

Arabidopsis thaliana (ecotype: Col-0) plants used for the ectopic expression experiments were grown on solid MS medium for about 10 days before being transferred to soil. All plants were incubated in a growth chamber at $22{ }^{\circ} \mathrm{C}$ and a photoperiod of 16-h light/8-h darkness.

\subsection{Isolation of the Full-Length IpASR cDNA}

A full-length cDNA library from I. pes-caprae was constructed and screened with the Full-length cDNA Over-eXpressor (FOX) gene hunting system using a yeast salt-sensitive mutant (AXT3) complementary assay approach (data not published). Thereafter, a full-length cDNA encoding the ASR protein (IpASR) that rescued the phenotype of AXT3 was selected and further investigated.

\subsection{Sequence Analysis of the IpASR Gene}

The full-length ASR cDNA sequence (GenBank accession no.: MF680587) was translated using the online ORFfinder translation tool (Available online: https:/ / www.ncbi.nlm.nih.gov / orffinder/). The ABA/WDS domain (Pfam entry PF02496) of the ASR protein was identified using the Pfam 31.0 server (Available online: http://pfam.xfam.org/). The 3D prediction of IpASR was also conducted with the online program Protein Fold Recognition Server tool ( PHYRE $^{2}$ ) (Available online: http://www.sbg.bio.ic.ac.uk/phyre2/html/page.cgi?id=index). MEGA 6 was used for the protein homology comparisons and phylogenetic reconstruction using the neighbor-joining (NJ) method [38]. Bootstrap values were estimated (with 1000 replicates) to assess the relative support for each branch. The IpASR protein was aligned with known plant ASRs using ClustalW software (Available online: http:/ / clustalw.ddbj.nig.ac.jp/). 
The genomic sequence of IpASR was also amplified with primer pairs IpASRPEF and IpASRPER (Supplementary Materials Table S2). The genomic DNA of I. pes-caprae was isolated with HiPure SF Plant DNA Kits (Magen, Guangzhou, China). The PCR product was inserted in pGEM T vector (Promega, Shanghai, China) and sequenced.

The protein sequences used were as follows: S. liaotungensis: SlASR (AGZ20206.1); S. brachiata: SbASR-1 (ACI15208.1); Camellia sinensis: CsASR (AHJ09608.1); Solanum chilense: ScASR (CBY05857.1); Mesembryanthemum crystallinum: McASR (AAC14177.1); Calystegia soldanella: CsoASR (BAB19963.1); Brachypodium distachyon: BdASR1 (XP_003565133.2); BdASR2 (XP_003567508.1); BdASR3 (XP_003567509.1); BdASR4 (XP_003577811.1); BdASR5 (KQJ82606.1); soybean: GmASR1 (NP_001336496.1); GmASR2 (NP_001237487.1); GmASR3 (XP_003536424.1); Ipomoea nil (morning glory): InASR (BAF46301.1); grape: VvASR (AAK69513.1); rice: OsASR1 (BAG88534.1); OsASR2 (BAS76319.1); OsASR3 (BAG89007.1); OsASR4 (XP_015633592.1); OsASR5 (BAG99580.1); OsASR6 (BAG87564.1); Maize: ZmASR1 (NP_001105361.2); ZmASR2 (NP_001278619.1); ZmASR3 (NP_001278619.1); ZmASR4 (NP_001152067.2); ZmASR5 (NP_001106235.1); ZmASR6 (XP_008645776.1); ZmASR7-1 (ONM32036.1); ZmASR7-2 (ONM32035.1); ZmASR7-3 (ONM32037.1); tomato: SIASR1 (Q08655.1); SIASR2 (P37219.1); SIASR3 (P37220.2); SIASR4 (AAY98032.1); SIASR5 (XP_004237807.1); apple: MdASR1 (XP_008340103.1); MdASR2 (XP_008358291.1); MdASR3 (XP_008348767.1); MdASR4 (XP_008381833.1); MdASR5 (XP_008381469.1).

\subsection{Bacterial Overexpression and Salt, Osmotic, Dehydration, and $\mathrm{H}_{2} \mathrm{O}_{2}$ Tolerance Assays in E. coli}

To further confirm the biological function of IpASR, the GST-IpASR fusion protein was inductively expressed in E. coli. The coding sequence (CDS) of IpASR was PCR-amplified using the primer pair (IpASREPF and IpASREPR) listed in Supplementary Materials Table S2. The PCR fragments were subsequently inserted into the BamHI site of pGEX 6p-1, followed by the GST-tag with the in-fusion technique (BD In-Fusion PCR cloning Kit, Takara Bio USA, Mountain View, CA, USA), thereby yielding the recombinant plasmid IpASR-pGEX 6p-1. The recombinant plasmid and empty vector pGEX 6p-1 (as a negative control) were then transformed into E. coli BL21 (DE3). A single colony was then inoculated in liquid Luria-Bertani (LB) medium and allowed to grow overnight at $37^{\circ} \mathrm{C}$ with constant shaking at $200 \mathrm{rpm}$. Inoculum (1\%) from the overnight-grown culture was added to fresh LB medium $(100 \mathrm{~mL})$ containing $100 \mu \mathrm{g} / \mathrm{mL}$ of ampicillin and allowed to grow at $37^{\circ} \mathrm{C}$ and $200 \mathrm{rpm}$. Expression was induced at an $\mathrm{OD}_{600}$ of 0.5 by $0.2 \mathrm{mM}$ isopropyl $\beta$-D-thiogalactopyranoside (IPTG), and the cells were allowed to grow at $30^{\circ} \mathrm{C}$ for $4 \mathrm{~h}$ with constant shaking at $200 \mathrm{rpm}$. The induced bacterial cells were harvested by centrifugation at $6000 \mathrm{rpm}$ for $10 \mathrm{~min}$ at $4{ }^{\circ} \mathrm{C}$, and the protein profiles were examined by $12 \%$ SDS PAGE.

A spot assay of E. coli was performed to test the stress tolerance of the IpASR protein, with three replicates for each sample. To evaluate salt, $\mathrm{H}_{2} \mathrm{O}_{2}$, and osmotic stress tolerance, cell cultures of $E$. coli (IPTG induced) containing pGEX 6p-1/IpASR-pGEX 6p-1 were adjusted to $\mathrm{OD}_{600} 1.0$ and then diluted serially (to 1:10, 1:100, and 1:1000). Two microliters of each sample was spotted onto the LB plates containing $0.2 \mathrm{mM}$ IPTG and the stress component ( $5 \%$ or $6 \% \mathrm{NaCl}, 2 \mathrm{M}$ sorbitol, 5 or $8 \mathrm{mM} \mathrm{H}_{2} \mathrm{O}_{2}$ ). For the drought tolerance test, $1 \mathrm{~mL}$ OD-adjusted cell cultures in tubes were immediately placed in a $40{ }^{\circ} \mathrm{C}$ drying oven and maintained there for $4 \mathrm{~h}$. Then, $100 \mu \mathrm{L}$ liquid LB medium was added to the samples, which were then maintained at $37^{\circ} \mathrm{C}$ for $1 \mathrm{~h}$ to recover. The samples were then diluted and spotted onto LB plates with $0.2 \mathrm{mM} \mathrm{IPTG}$. The plates were incubated at $37^{\circ} \mathrm{C}$ for $10 \mathrm{~h}$. The bacterial colonies were counted (colony former unit, $\mathrm{CFU}$ ) and the differences were analyzed.

We also performed a growth curve assay of recombinant $E$. coli in liquid LB culture to further confirm the functionality of IpASR. Briefly, $1 \mathrm{~mL}$ inoculum $\left(\mathrm{OD}_{600}\right.$ value 1.0) was added to $10 \mathrm{~mL} \mathrm{LB}$ medium (supplied with $0.2 \mathrm{mM} \mathrm{IPTG)} \mathrm{containing} \mathrm{salt} \mathrm{(} 3 \%$ or $4 \% \mathrm{NaCl}$ ), sorbitol $(0.8$ or $1 \mathrm{M})$, or $\mathrm{H}_{2} \mathrm{O}_{2}$ $(0.7$ or $0.9 \mathrm{mM})$ and incubated at $37^{\circ} \mathrm{C}$ with shaking $(180 \mathrm{rpm})$. The aliquots were removed from each treatment every $2 \mathrm{~h}$ from 2 to $12 \mathrm{~h}$ and absorbance $\left(\mathrm{OD}_{600}\right)$ was measured. Abiotic stress (salt, osmotic 
and $\mathrm{H}_{2} \mathrm{O}_{2}$ ) tolerances were determined with respect to the control cultures (bacterial cells and empty vector pGEX 6p-1 controls).

\subsection{Subcellular Localization Analysis}

The CDS (without a termination codon) of the IpASR gene was amplified by PCR using the primer pair IpASRGF and IpASRGR (Supplementary Materials Table S2). The PCR product was inserted into the BamHI site of the pUC/GFP vector to generate an IpASR-GFP in-frame fusion protein, following the in-fusion technique (Clontech, Mountain View, CA, USA), yielding the recombinant plasmid IpASR-pUC/GFP. After sequencing confirmation, the fusion construct and negative control (empty vector) were co-transfected into protoplasts $\left(3 \times 10^{4}\right.$ protoplasts $)$ using polyethylene glycol (PEG)-calcium solution (0.4 g. $\mathrm{mL}^{-1}$ PEG 4000, $0.2 \mathrm{M}$ mannitol, $0.1 \mathrm{M} \mathrm{CaCl}_{2}$ ). After washing and resuspending in W5 solution ( $154 \mathrm{mM} \mathrm{NaCl}, 125 \mathrm{mM} \mathrm{CaCl}_{2}, 5 \mathrm{mM} \mathrm{KCl}, 5 \mathrm{mM}$ glucose, $2 \mathrm{mM}$ MES, mesophyll protoplasts were incubated under white light for 12-18 h. Green fluorescent protein (GFP) fluorescence was visualized using a confocal laser scanning microscope (LSM, 510 META, Zeiss, Jena, Germany). The NLS-mCherry construction was co-transformed with the construction or empty vector as a nuclear localized marker.

\subsection{Analysis of Transcriptional Activities in Yeast}

The full-length CDS of IpASR and three truncated IpASRs were cloned into vector pGBKT7 (Clontech, Mountain View, CA, USA) for transcription activation analysis. These constructs along with the negative control pGBKT7 and pGBKT7-Lam plasmids, or positive control pGBKT7-53 plasmid, were transformed into Saccharomyces cerevisiae strain AH109 using the lithium acetate mediated method according to the Yeast Protocols Handbook (Clontech, Mountain View, CA, USA), and the vector PGADT7-T was co-transformed with all of the above constructs and positive/negative contorls (pGBKT7-53, pGBKT7, and pGBKT7-Lam). The yeast clones was cultured in liquid SD-2 medium to $\mathrm{OD}_{600}$ until 1.0, after which they were diluted using a gradient dilution (1:10, 1:100, and 1:1000). Two-microliter yeast cultures were spotted onto the corresponding synthetically defined (SD/-Trp/-Leu and SD/-Trp/-Leu/-His) medium plates for 2 days at $30{ }^{\circ} \mathrm{C}$. Yeast transformation and determination of blue/white colonies were conducted according to the instructions of the manufacturer (Clontech), and X- $\alpha-\mathrm{Gal}$ was used as a substrate for the reporter gene MEL1. Primers used for plasmid construction are shown in Supplementary Materials Table S2.

\subsection{Expression Pattern Analysis}

Total RNA was isolated from the I. pes-caprae tissues using HiPure Plant RNA Kits (Magen, Guangzhou, China), and the cDNA was synthesized by TransScript One-Step gDNA Removal and cDNA Synthesis SuperMix (TransGen Biotech, Beijing, China) according to the manufacturer's instructions. Quantitative reverse transcript PCR (qRT-PCR) was performed to examine the expression levels of IPASR in the various tissues of the seedling and adult I. pes-caprae plants, including seedling roots, seedling leaves, buds, mature roots, vines, mature leaves, flower buds, petals, and young seeds. The I. pes-caprae seedling samples (roots, vines, and leaves), treated with salt, simulated drought or osmotic stress, oxidative stress, freezing stress, and ABA were also assessed to examine the expression changes of IpASR. All of the gene expression data obtained via qRT-PCR were normalized to the expression of IpUBQ (GenBank accession number: MF502417). The primers used for qRT-PCR are listed in Supplementary Materials Table S2.

For detection of the expression of antioxidative related genes (CAT1, CAT2, CSD1, and FSD1) in Arabidopsis (wild-type WT or transgenic plants), total RNA was isolated from the rosette leaves at different time points (with or without treatment), and cDNA synthesis was performed as per the above procedure. The reference gene for the RT-PCR was ACT2 (At3g18780) in Arabidopsis. The primers used for qRT-PCR are listed in Supplementary Materials Table S2. 


\subsection{Generation of Transgenic Arabidopsis}

To generate the recombinant vector for the overexpression assay in transgenic Arabidopsis, the full-length cDNA of IpASR was PCR-amplified using the primer pair IpASROXF and IpASROXR (Supplementary Materials Table S2). The PCR product was cloned into the BamHI site of the pBIm plasmid [39] to generate IpASR-pBIm with an expression cassette under the control of the CaMV 35S promoter. After sequencing confirmation, the construct was transferred into Agrobacterium tumefaciens GV3101 and then transformed into Arabidopsis using the floral-dip method. Seeds of the T1 and T2 generations were screened on MS agar medium containing $50 \mathrm{mg} / \mathrm{L}$ kanamycin. Positive transgenic plants were selected according to the segregation ratio (resistant:sensitive $=3: 1$ ) and confirmed by genomic PCR with the primer pairs IpASROXF/IpASROXR. qRT-PCR and RT-PCR were also performed with primer pairs ASRF/ASRR and AtAct2F/AtAct2R (Supplementary Materials Table S2) in order to identify the expression level of IpASR in transgenic Arabidopsis.

\subsection{Stress Tolerance of Transgenic Arabidopsis}

For the salt and osmotic stress tolerance assay, transgenic Arabidopsis T3 (IpASR OX3 and IpASR $O X 8)$ and WT were used. Sterilized seeds were germinated in MS medium under a 16-h photoperiod cycle at $25{ }^{\circ} \mathrm{C}$, and 4-day-old seedlings were transplanted into medium supplemented with 100, 125, and $150 \mathrm{mM} \mathrm{NaCl}$ or 200, 300 and 400 mannitol. The germination rate and root length were measured. In brief, the seed germination rate of IpASR transgenic Arabidopsis was detected under $\mathrm{NaCl}(100$, $125,150,175$ and $200 \mathrm{mM})$ and mannitol $(200,300$ and $400 \mathrm{mM})$ challenges on MS plates to assess whether the overexpression of IpASR could improve the salt and osmotic tolerance of the transgenic Arabidopsis seeds. The root length was also calculated to evaluate the influence of IpASR on the transgenic Arabidopsis seedlings under abiotic stress. The WT Arabidopsis was used as a control.

For salt and drought tolerance assays, 30 one-week-old seedlings of each genotype (IpASR OX3, IpASR OX8, and WT) were planted in sieve-like pots and watered well for two weeks. Prior to the salt/drought experiments, the plants of each genotype were cultured in a growth chamber as described above without watering for 10 days in order to ensure that the surrounding growing environment was devoid of excess water. The plants were then subjected to the following assays. For the drought tolerance assays, the WT and transgenic plants (IpASR OX3 and IpASR OX8) were maintained under drought conditions for 9 days, and were then re-watered for 7 days. For the salt tolerance assays, the plants of each genotype were planted in sieve-like pots and watered well, as described for the drought tolerance treatment. Water was withheld for $10 \mathrm{~d}$ before irrigating with $\mathrm{NaCl}$ solution (150 and $200 \mathrm{mM}$ ) from the bottom of the plates. When the soil was completely saturated with salt water, the $\mathrm{NaCl}$ solution was removed and the plants were cultured normally. The plants were grown in the salt-saturated soil for 10 days and then re-watered for 7 days. The survival rate of the Arabidopsis plants was measured once the entire assay was complete.

For the oxidative stress analyses of the transgenic overexpression lines and WT plants, 3-week-old seedlings of IpASR OXs and WT Arabidopsis plants in the soil were sprayed evenly with $20 \mu \mathrm{M}$ and $50 \mu \mathrm{M}$ methyl viologen (MV) for two times (first plants were sprayed for 3 time at beginning each pot, then second session plants were sprayed for 12 time on the 7 th day each pot, one spray $\approx 100 \mu \mathrm{L}$ ), after which the plants were cultured normally for 14 days. The phenotype was recorded.

\subsection{Physiological and Histochemical Analysis}

The relative water content (RWC) of adult Arabidopsis plants was determined as described according to $\mathrm{Hu}$ et al. [28]. Fresh weight (FW) of rosette leaves were recorded followed by soaking the leaves for $4 \mathrm{~h}$ in distilled water at room temperature with constant light. After this treatment the turgid weight (TW) was recorded. The leaves were then dried for $24 \mathrm{~h}$ at $80{ }^{\circ} \mathrm{C}$ to obtain the total dry weight (DW). RWC was calculated from the equation: RWC $(\%)=[(\mathrm{FW}-\mathrm{DW}) /(\mathrm{TW}-\mathrm{DW})] \times 100 \%$. 
Rosette leaves were collected after each treatment, and IL (ion leakage) was measured with a DDS-307A conductivity meter (Shanghai Jingke, Shanghai, China), according to Hu et al. [28] with slight modifications. Arabidopsis rosette leaves were incubated in $20 \mathrm{~mL}$ double-distilled water at room temperature with occasional shaking for $12 \mathrm{~h}$. Initial conductivity (C1) was measured with a conductivity meter followed by boiling of the samples for $30 \mathrm{~min}$ to measure complete IL. The leaves were then cooled to room temperature to measure the electrolyte conductivity (C2). IL was calculated according to the equation: IL $(\%)=\mathrm{C} 1 / \mathrm{C} 2 \times 100 \%$.

Free proline content, malondialdehyde (MDA) content, and superoxide dismutase (SOD) and catalase (CAT) activities were determined using proline, MDA, SOD, and Catalase Assay Kits, according to the manufacturer's instructions, respectively (Nanjing Jiancheng, Nanjing, China). In situ detection of $\mathrm{H}_{2} \mathrm{O}_{2}$ and $\mathrm{O}_{2}{ }^{-}$was determined with $1 \mathrm{mg} / \mathrm{mL}$ nitro-blue tetrazolium (NBT) or $1 \mathrm{mg} / \mathrm{mL} 3,3^{\prime}$-diaminobenzidine (DAB) solution for $12 \mathrm{~h}$ and clearing in $96 \%$ ethanol, respectively, as previously described [40].

\subsection{Statistical Analysis}

All the experiments in this study were repeated three times independently, and the data shown are the mean $\pm \mathrm{SD}(n \geq 3)$. Statistical analyses were performed using the statistical tools (Student's $t$-test) of Microsoft Excel (Microsoft Corporation, Albuquerque, NM, USA).

Supplementary Materials: The following are available online at http:/ /www.mdpi.com/1422-0067/19/8/2252/s1.

Author Contributions: Conceptualization, M.Z.; Data curation, M.Z., J.-X.Z., H.Z. and H.-X.S.; Formal analysis, H.Z.; Funding acquisition, M.Z., K.-F.X. and S.-G.J.; Methodology, J.-X.Z. and H.Z.; Project administration, K.-F.X. and M.Z.; Supervision, S.-G.J. and M.Z.; Writing—original draft, M.Z.; Writing-review \& editing, M.Z.

Acknowledgments: This research was supported by grants from the National Natural Sciences Foundation of China (31570257 and 31271702), the National Key Research and Development Program of China (2016YFC1403002), the 'Strategic Priority Research Program' of the Chinese Academy of Sciences (XDA13020500), and "the Twelfth Five-Year" National S\&T Projects for Rural Areas (2015BAL04B04). The funders had no roles in the study design, data collection and analysis, decision to publish, or preparation of the manuscript.

Conflicts of Interest: The authors declare that the research was conducted in the absence of any commercial or financial relationships that could be construed as a potential conflict of interest.

$\begin{array}{ll}\text { Abbreviations } \\ \text { ASR } & \text { Abscisic acid, Stress and Ripening } \\ \text { ABA/WDS } & \text { Abscisic acid/Water Deficit Stress } \\ \text { LEA } & \text { Late Embryogenesis Abundant } \\ \text { ORF } & \text { Open Reading Frame } \\ \text { GRAVY } & \text { Grand Average of Hydropathicity } \\ \text { IDP } & \text { Intrinsically Disordered Protein } \\ \text { NLS } & \text { Nuclear Localization Signal } \\ \text { MV } & \text { Methyl Viologen } \\ \text { IPTG } & \text { Isopropyl } \beta \text {-D-thiogalactopyranoside } \\ \text { LB } & \text { Luria-Bertani } \\ \text { GFP } & \text { Green Fluorescent Protei } \\ \text { MS } & \text { Murashige and Skoog } \\ \text { ROS } & \text { Reactive Oxygen Species } \\ \text { SDS PAGE } & \text { Sodium Dodecyl Sulfate Polyacrylamide Gel Electropheresis } \\ \text { qRT-PCR } & \text { Quantitative Reverse Transcript Polymerase Chain Reaction } \\ \text { OX } & \text { Over eXpression } \\ \text { WT } & \text { Wild Type } \\ \text { MDA } & \text { Malondialdehyde } \\ \text { IL } & \text { Ion Leakage } \\ \text { RWC } & \text { Relative Water Content } \\ \text { SOD } & \text { Superoxide Dismutas } \\ \text { CAT } & \text { Catalase }\end{array}$




\section{References}

1. Hanin, M.; Ebel, C.; Ngom, M.; Laplaze, L.; Masmoudi, K. New insights on plant salt tolerance mechanisms and their potential use for breeding. Front. Plant Sci. 2016, 7, 1787. [CrossRef] [PubMed]

2. Ahanger, M.A.; Akram, N.A.; Ashraf, M.; Alyemeni, M.N.; Wijaya, L.; Ahmad, P. Plant responses to environmental stresses-From gene to biotechnology. AoB Plants 2017, 9, plx025. [CrossRef] [PubMed]

3. Carrari, F.; Fernie, A.R.; Iusem, N.D. Heard it through the grapevine? ABA and sugar cross-talk: The ASR story. Trends Plant Sci. 2004, 9, 57-59. [CrossRef] [PubMed]

4. Dominguez, P.G.; Carrari, F. ASR1 transcription factor and its role in metabolism. Plant Signal Behav. 2015, 10, e992751. [CrossRef] [PubMed]

5. Battaglia, M.; Olvera-Carrillo, Y.; Garciarrubio, A.; Campos, F.; Covarrubias, A.A. The enigmatic LEA proteins and other hydrophilins. Plant Physiol. 2008, 148, 6-24. [CrossRef] [PubMed]

6. Iusem, N.D.; Bartholomew, D.M.; Hitz, W.D.; Scolnik, P.A. Tomato (Lycopersicon esculentum) transcript induced by water deficit and ripening. Plant Physiol. 1993, 102, 1353-1354. [CrossRef] [PubMed]

7. González, R.M.; Iusem, N.D. Twenty years of research on Asr (ABA-stress-ripening) genes and proteins. Planta 2014, 239, 941-949. [CrossRef] [PubMed]

8. Golan, I.; Dominguez, P.G.; Konrad, Z.; Shkolnik-Inbar, D.; Carrari, F.; Bar-Zvi, D. Tomato ABSCISIC ACID STRESS RIPENING (ASR) gene family revisited. PLoS ONE 2014, 9, e107117. [CrossRef] [PubMed]

9. Wang, L.; Hu, W.; Feng, J.; Yang, X.; Huang, Q.; Xiao, J.; Liu, Y.; Yang, G.; He, G. Identification of the ASR gene family from Brachypodium distachyon and functional characterization of BdASR1 in response to drought stress. Plant Cell Rep. 2016, 35, 1221-1234. [CrossRef] [PubMed]

10. Huang, K.; Zhong, Y.; Li, Y.; Zheng, D.; Cheng, Z.M. Genome-wide identification and expression analysis of the apple ASR gene family in response to Alternaria alternata f. sp. Mali. Genome 2016, 59, 866-878. [CrossRef] [PubMed]

11. Golldack, D.; Li, C.; Mohan, H.; Probst, N. Tolerance to drought and salt stress in plants: Unraveling the signaling networks. Front. Plant Sci. 2014, 5, 151. [CrossRef] [PubMed]

12. Dai, J.R.; Liu, B.; Feng, D.R.; Liu, H.Y.; He, Y.M.; Qi, K.B.; Wang, H.B.; Wang, J.F. MpAsr encodes an intrinsically unstructured protein and enhances osmotic tolerance in transgenic Arabidopsis. Plant Cell Rep. 2011, 30, 1219-1230. [CrossRef] [PubMed]

13. Jha, B.; Lal, S.; Tiwari, V.; Yadav, S.K.; Agarwal, P.K. The SbASR-1 gene cloned from an extreme halophyte Salicornia brachiata enhances salt tolerance in transgenic tobacco. Mar. Biotechnol. 2012, 14, 782-792. [CrossRef] [PubMed]

14. Tiwari, V.; Chaturvedi, A.K.; Mishra, A.; Jha, B. Introgression of the SbASR-1 gene cloned from a halophyte Salicornia brachiate enhances salinity and drought endurance in transgenic groundnut (Arachis hypogaea) and acts as a transcription factor. PLOS ONE 2015, 10, e0131567. [CrossRef] [PubMed]

15. Hu, Y.X.; Yang, X.; Li, X.L.; Yu, X.D.; Li, Q.L. The SlASR gene cloned from the extreme halophyte Suaeda liaotungensis K. enhances abiotic stress tolerance in transgenic Arabidopsis thaliana. Gene 2014, 549, $243-251$. [CrossRef] [PubMed]

16. Li, J.; Dong, Y.; Li, C.; Pan, Y.; Yu, J. SiASR4, the target gene of SiARDP from Setaria italica, improves abiotic stress adaption in plants. Front. Plant Sci. 2017, 7, 2053. [CrossRef] [PubMed]

17. Ou-Yang, P.Y.; Liu, N.; Zhang, W.W.; Wang, J.; Jian, S.G. Biological and eco-physiological characteristics of a beach plant ipomoea pescaprae. J. Hunan Univ. Sci. Technol. Nat. Sci. Ed. 2011, 26, 117-121.

18. Mishra, A.; Tanna, B. Halophytes: Potential resources for salt stress tolerance genes and promoters. Front. Plant Sci. 2017, 8, 829. [CrossRef] [PubMed]

19. Shabala, S. Learning from halophytes: Physiological basis and strategies to improve abiotic stress tolerance in crops. Ann. Bot. 2013, 112, 1209-1221. [CrossRef] [PubMed]

20. Yuan, H.M.; Sheng, Y.; Chen, W.J.; Lu, Y.Q.; Tang, X.; Ou-Yang, M.; Huang, X. Overexpression of Hevea brasiliensis HbICE1 enhances cold tolerance in Arabidopsis. Front. Plant Sci. 2017, 8, 1462. [CrossRef] [PubMed]

21. Ben, R.K.; Abdelly, C.; Savouré, A. How reactive oxygen species and proline face stress together. Plant Physiol. Biochem. 2014, 80, 278-284.

22. You, J.; Chan, Z. ROS regulation during abiotic stress responses in crop plants. Front. Plant Sci. 2015, 6, 1092. [CrossRef] [PubMed] 
23. Han, H.J.; Peng, R.H.; Zhu, B.; Fu, X.Y.; Zhao, W.; Shi, B.; Yao, Q.H. Gene expression profiles of Arabidopsis under the stress of methyl viologen: A microarray analysis. Mol. Biol. Rep. 2014, 41, 7089-7102. [CrossRef] [PubMed]

24. Padaria, J.C.; Yadav, R.; Tarafdar, A.; Lone, S.A.; Kumar, K.; Sivalingam, P.N. Molecular cloning and characterization of drought stress responsive abscisic acid-stress-ripening (Asr1) gene from wild jujube, Ziziphus nummularia (Burm.f.) Wight \& Arn. Mol. Biol. Rep. 2016, 43, 849-859. [PubMed]

25. Sun, P.; Miao, H.; Yu, X.; Jia, C.; Liu, J.; Zhang, J.; Wang, J.; Wang, Z.; Wang, A.; Xu, B.; et al. A novel role for banana MaASR in the regulation of flowering time in transgenic Arabidopsis. PLoS ONE 2016, 11, e0160690. [CrossRef] [PubMed]

26. Canel, C.; Bailey-Serres, J.N.; Roose, M.L. Pummelo fruit transcript homologous to ripening-induced genes. Plant Physiol. 1995, 108, 1323-1324. [CrossRef] [PubMed]

27. Padmanabhan, V.; Dias, D.M.; Newton, R.J. Expression analysis of a gene family in loblolly pine (Pinus taeda L.) induced by water deficit stress. Plant Mol. Biol. 1997, 35, 801-807. [CrossRef] [PubMed]

28. Hu, W.; Huang, C.; Deng, X.; Zhou, S.; Chen, L.; Li, Y.; Wang, C.; Ma, Z.; Yuan, Q.; Wang, Y.; et al. TaASR1, a transcription factor gene in wheat, confers drought stress tolerance in transgenic tobacco. Plant Cell Environ. 2013, 36, 1449-1464. [CrossRef] [PubMed]

29. Yamada, T.; Ichimura, K.; Kanekatsu, M.; van Doorn, W.G. Gene expression in opening and senescing petals of morning glory (Ipomoea nil) flowers. Plant Cell Rep. 2007, 26, 823-835. [CrossRef] [PubMed]

30. Philippe, R.; Courtois, B.; McNally, K.L.; Mournet, P.; El-Malki, R.; le Paslier, M.C.; Fabre, D.; Billot, C.; Brunel, D.; Glaszmann, J.C.; et al. Structure, allelic diversity and selection of Asr genes, candidate for drought tolerance, in Oryza sativa L. and wild relatives. Theor. Appl. Genet. 2010, 121, 769-787. [CrossRef] [PubMed]

31. Cortés, A.J.; Chavarro, M.C.; Madriñán, S.; This, D.; Blair, M.W. Molecular ecology and selection in the drought-related Asr gene polymorphisms in wild and cultivated common bean (Phaseolus vulgaris L.). BMC Genet. 2012, 13, 58. [CrossRef] [PubMed]

32. Kim, I.S.; Kim, Y.S.; Yoon, H.S. Rice ASR1 protein with reactive oxygen species scavenging and chaperone-like activities enhances acquired tolerance to abiotic stresses in Saccharomyces cerevisiae. Mol. Cells 2012, 33, 285-293. [CrossRef] [PubMed]

33. Arenhart, R.A.; Schunemann, M.; Bucker Neto, L.; Margis, R.; Wang, Z.Y.; Margis-Pinheiro, M. Rice ASR1 and ASR5 are complementary transcription factors regulating aluminium responsive genes. Plant Cell Environ. 2016, 39, 645-651. [CrossRef] [PubMed]

34. Li, R.H.; Liu, G.B.; Wang, H.; Zheng, Y.Z. Effects of $\mathrm{Fe}^{3+}$ and $\mathrm{Zn}^{2+}$ on the structural and thermodynamic properties of a Soybean ASR protein. Biosci. Biotechnol. Biochem. 2013, 77, 475-481. [CrossRef] [PubMed]

35. Lippuner, V.; Cyert, M.S.; Gasser, C.S. Two classes of plant cDNA clones differentially complement yeast calcineurin mutants and increase salt tolerance of wild-type yeast. J. Biol. Chem. 1996, 271, 12859-12866. [CrossRef] [PubMed]

36. Rigó, G.; Valkai, I.; Faragó, D.; Kiss, E.; van Houdt, S.; van de Steene, N.; Hannah, M.A.; Szabados, L. Gene mining in halophytes: Functional identification of stress tolerance genes in Lepidium crassifolium. Plant Cell Environ. 2016, 39, 2074-2084. [CrossRef] [PubMed]

37. Virlouvet, L.; Jacquemot, M.P.; Gerentes, D.; Corti, H.; Bouton, S.; Gilard, F.; Valot, B.; Trouverie, J.; Tcherkez, G.; Falque, M.; et al. The ZmASR1 protein influences branched-chain amino acid biosynthesis and maintains kernel yield in maize under water-limited conditions. Plant Physiol. 2011, 157, 917-936. [CrossRef] [PubMed]

38. Tamura, K.; Stecher, G.; Peterson, D.; Filipski, A.; Kumar, S. MEGA6: Molecular evolutionary genetics analysis version 6.0. Mol. Biol. Evol. 2013, 30, 2725-2729. [CrossRef] [PubMed]

39. Yang, L.X.; Wang, R.Y.; Ren, F.; Liu, J.; Cheng, J.; Lu, Y.T. AtGLB1 enhances the tolerance of Arabidopsis to hydrogen peroxide stress. Plant Cell Physiol. 2005, 46, 1309-1316. [CrossRef] [PubMed]

40. Cao, Y.; Xiang, X.; Geng, M.; You, Q.; Huang, X. Effect of HbDHN1 and HbDHN2 genes on abiotic stress responses in arabidopsis. Front. Plant Sci. 2017, 8, 470. [CrossRef] [PubMed]

(C) 2018 by the authors. Licensee MDPI, Basel, Switzerland. This article is an open access article distributed under the terms and conditions of the Creative Commons Attribution (CC BY) license (http:/ / creativecommons.org/licenses/by/4.0/). 